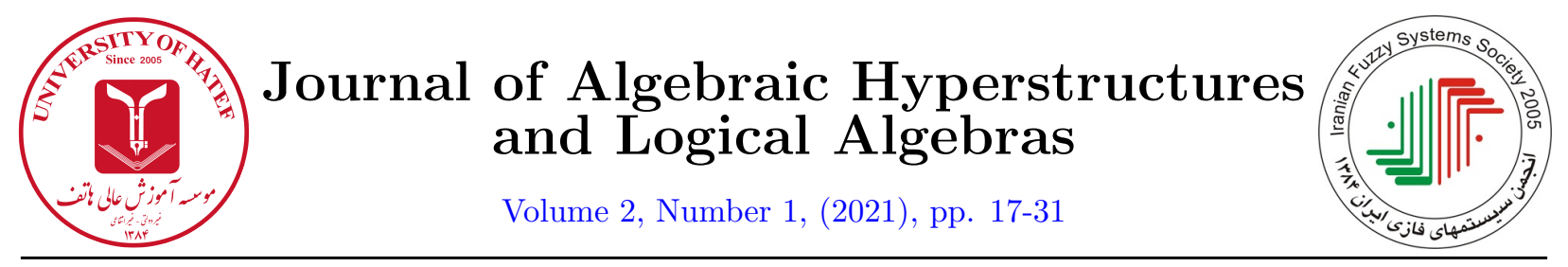

\title{
Crossing cubic ideals of BCK/BCI-algebras
}

\author{
Y.B. Jun $^{1}$ and S.Z. Song ${ }^{2}$ \\ ${ }^{1}$ Department of Mathematics Education, Gyeongsang National University, Jinju 52828, Korea \\ ${ }^{2}$ Department of Mathematics, Jeju National University, Jeju 63243, Korea
}

skywine@gmail.com; szsong@jejunu.ac.kr

\begin{abstract}
The notions of a crossing cubic ideal in a BCK/BCI-algebra, a closed crossing cubic ideal in a BCI-algebra, and a crossing cubic o-subalgebra of a BCK-algebra with the condition (S) are introduced, and several properties are investigated. The relationship between them is established. Conditions for a crossing cubic structure to be a closed crossing cubic ideal are provided. Conditions under which crossing cubic ideals are closed are explored. Characterizations of crossing cubic ideals are discussed. The translation of crossing cubic subalgebras and crossing cubic ideals are studied. Conditions for the translation of a crossing cubic structure to be a crossing cubic subalgebra (ideal) are provided, and its characterization is established.
\end{abstract}

\section{Article Information}

Corresponding Author:

Y.B. Jun;

Received: February 2021;

Accepted: Invited paper;

Papertype: Original.

Keywords:

Crossing cubic o-subalgebra, (closed) crossing cubic ideal, 0 -crossing cubic structure, translation.

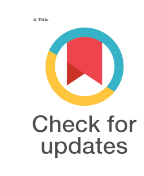

\section{Introduction}

In a universe $M$, the (crisp) set $B$ can be defined as the characteristic function $\mu_{B}: M \rightarrow\{0,1\}$ where 0 and 1 correspond to elements belonging to the set $B$ and those excluded from the set $B$, respectively. If we take an extension $[0,1]$ of the range $\{0,1\}$ in the characteristic function $\mu_{B}: M \rightarrow\{0,1\}$, we can get a new function from $M$ into $[0,1]$ which is called the fuzzy set, and it is introduced by Zadeh in the paper [9]. The degree of membership of an element in a traditional fuzzy set spans the interval $[0,1]$. Thus, fuzzy sets are a very useful tool for dealing with positive information. But there is a limit to dealing with negative information. In real life, both positive and negative information coexist. So we feel that we need mathematical tools to deal with negative information. From this point of view, Jun et al. 4] introduced a new function called a negative value function and applied it to BCK/BCI-algebras. As an extension of fuzzy sets, Zadeh [10] made a fuzzy set with in interval-valued membership function so called an interval-valued fuzzy set (briefly, IVF set). Using the notion of $\mathcal{N}$-functions and IVF sets, Jun et al. [3] established an extension of a bipolar-valued fuzzy set, which is introduced by Lee [5]. They called it a crossing cubic structure, and investigated several properties. They defined the same direction order and the opposite direction order in crossing cubic structures. Also, they define S-union, S-intersection, O-union and O-intersection of crossing cubic structures, and discussed their related properties. They applied crossing cubic structures to BCK/BCI-algebras, and studied crossing cubic subalgebras.

https://doi.org/10.52547/HATEF.JAHLA.2.1.2 
In this article, we introduce the notions of a crossing cubic ideal in a BCK/BCI-algebra, a closed crossing cubic ideal in a BCI-algebra, and a crossing cubic o-subalgebra of a BCK-algebra with the condition (S). We identify the relationship between them. We provide conditions for a crossing cubic structure to be a closed crossing cubic ideal We explore the conditions under which crossing cubic ideals are closed. We discuss characterizations of crossing cubic ideals. We study the translation of crossing cubic subalgebras and crossing cubic ideals. We find conditions for the translation of a crossing cubic structure to be a crossing cubic subalgebra (ideal), and consider its characterization.

\section{Preliminaries}

\subsection{Basic concepts about BCK/BCI-algebras}

A set $M$ with a binary operation " $\rightsquigarrow$ " and a special element 0 is called a BCI-algebra if it satisfies:

$$
\begin{aligned}
& \left(\forall \kappa_{1}, \kappa_{2}, \kappa_{3} \in M\right)\left(\left(\left(\kappa_{1} \rightsquigarrow \kappa_{2}\right) \rightsquigarrow\left(\kappa_{1} \rightsquigarrow \kappa_{3}\right)\right) \rightsquigarrow\left(\kappa_{3} \rightsquigarrow \kappa_{2}\right)=0\right), \\
& \left(\forall \kappa_{1}, \kappa_{2} \in M\right)\left(\left(\kappa_{1} \rightsquigarrow\left(\kappa_{1} \rightsquigarrow \kappa_{2}\right)\right) \rightsquigarrow \kappa_{2}=0\right), \\
& \left(\forall \kappa_{1} \in M\right)\left(\kappa_{1} \rightsquigarrow \kappa_{1}=0\right), \\
& \left(\forall \kappa_{1}, \kappa_{2} \in M\right)\left(\kappa_{1} \rightsquigarrow \kappa_{2}=0, \kappa_{2} \rightsquigarrow \kappa_{1}=0 \Rightarrow \kappa_{1}=\kappa_{2}\right) .
\end{aligned}
$$

A BCI-algebra $M$ satisfying the following condition:

$$
\left(\forall \kappa_{1} \in M\right)\left(0 \rightsquigarrow \kappa_{1}=0\right)
$$

is called a $B C K$-algebra.

Every BCK/BCI-algebra $M$ satisfies:

$$
\begin{aligned}
& \left(\forall \kappa_{1} \in M\right)\left(\kappa_{1} \rightsquigarrow 0=\kappa_{1}\right), \\
& \left(\forall \kappa_{1}, \kappa_{2}, \kappa_{3} \in M\right)\left(\left(\kappa_{1} \rightsquigarrow \kappa_{2}\right) \rightsquigarrow \kappa_{3}=\left(\kappa_{1} \rightsquigarrow \kappa_{3}\right) \rightsquigarrow \kappa_{2}\right), \\
& \left(\forall \kappa_{1}, \kappa_{2}, \kappa_{3} \in M\right)\left(\kappa_{1} \leq \kappa_{2} \Rightarrow \kappa_{1} \rightsquigarrow \kappa_{3} \leq \kappa_{2} \rightsquigarrow \kappa_{3}, \kappa_{3} \rightsquigarrow \kappa_{2} \leq \kappa_{3} \rightsquigarrow \kappa_{1}\right),
\end{aligned}
$$

Every BCI-algebra $M$ satisfies:

$$
\begin{aligned}
& \left(\forall \kappa_{1}, \kappa_{2} \in M\right)\left(\kappa_{1} \rightsquigarrow\left(\kappa_{1} \rightsquigarrow\left(\kappa_{1} \rightsquigarrow \kappa_{2}\right)\right)=\kappa_{1} \rightsquigarrow \kappa_{2}\right), \\
& \left(\forall \kappa_{1}, \kappa_{2} \in M\right)\left(0 \rightsquigarrow\left(\kappa_{1} \rightsquigarrow \kappa_{2}\right)=\left(0 \rightsquigarrow \kappa_{1}\right) \rightsquigarrow\left(0 \rightsquigarrow \kappa_{2}\right)\right) .
\end{aligned}
$$

A subset $L$ of a BCK/BCI-algebra $M$ is called

- a subalgebra of $M$ (see [2, 6]) if $\kappa_{1} \rightsquigarrow \kappa_{2} \in L$ for all $\kappa_{1}, \kappa_{2} \in L$.

- an ideal of $M$ (see [2, 6]) if it satisfies:

$$
\begin{aligned}
& 0 \in L, \\
& \left(\forall \kappa_{1}, \kappa_{2} \in M\right)\left(\kappa_{1} \rightsquigarrow \kappa_{2} \in L, \kappa_{2} \in L \Rightarrow \kappa_{1} \in L\right) .
\end{aligned}
$$

A BCK-algebra $M$ is said to have condition (S) (see [6]) if given $a, b \in M$ the set

$$
M(a, b):=\left\{\kappa_{1} \in M \mid \kappa_{1} \rightsquigarrow a \leq b\right\}
$$

has a greatest element, say $a \circ b$.

A subset $L$ of a BCK-algebra $M$ with condition (S) is called a o-subalgebra of $M$ (see [6]) if $a \circ b \in L$ for all $a, b \in L$.

For more information on BCK/BCI-algebra, please refer to the books [2] and [6]. 


\subsection{Basic concepts about crossing cubic structures}

The collection of all functions from a set $M$ to $[-1,0]$ is denoted by $\mathcal{F}(M,[-1,0])$, and an element of $\mathcal{F}(X,[-1,0])$ is called a negative-valued function from $M$ to $[-1,0]$ (briefly, $\mathcal{N}$-function on $M$ ). Define a relation $\leq$ on $\mathcal{F}(M,[-1,0])$ as follows:

$$
\xi \leq \eta \Leftrightarrow\left(\forall \kappa_{1} \in M\right)\left(\xi\left(\kappa_{1}\right) \leq \eta\left(\kappa_{1}\right)\right)
$$

for all $\xi, \eta \in \mathcal{F}(M,[-1,0])$.

An interval number is defined to be a subinterval $\tilde{c}=\left[c^{-}, c^{+}\right]$of $[0,1]$, where $0 \leq c^{-} \leq c^{+} \leq 1$. The interval numbers $[0,0]$ and $[1,1]$ are denoted by $\tilde{0}$ and $\tilde{1}$, respectively. The set of all interval numbers is denoted by $[[0,1]]$, and we define the "refined minimum" (briefly, "rmin") of two interval numbers. We

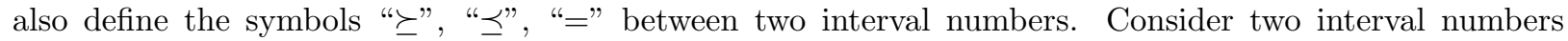
$\tilde{c}_{1}:=\left[c_{1}^{-}, c_{1}^{+}\right]$and $\tilde{c}_{2}:=\left[c_{2}^{-}, c_{2}^{+}\right]$. Then

$$
\begin{aligned}
& \operatorname{rmin}\left\{\tilde{c}_{1}, \tilde{c}_{2}\right\}:=\left[\min \left\{c_{1}^{-}, c_{2}^{-}\right\}, \min \left\{c_{1}^{+}, c_{2}^{+}\right\}\right], \\
& \tilde{c}_{1} \succeq \tilde{c}_{2} \Leftrightarrow c_{1}^{-} \geq c_{2}^{-}, c_{1}^{+} \geq c_{2}^{+},
\end{aligned}
$$

and similarly we may have $\tilde{c}_{1} \preceq \tilde{c}_{2}$ and $\tilde{c}_{1}=\tilde{c}_{2}$. To say $\tilde{c}_{1} \succ \tilde{c}_{2}$ (resp. $\left.\tilde{c}_{1} \prec \tilde{c}_{2}\right)$ we mean $\tilde{c}_{1} \succeq \tilde{c}_{2}$ and $\tilde{c}_{1} \neq \tilde{c}_{2}$ (resp. $\tilde{c}_{1} \preceq \tilde{c}_{2}$ and $\left.\tilde{c}_{1} \neq \tilde{c}_{2}\right)$. Let $\tilde{c}_{i} \in[[0,1]]$ where $i \in \Lambda$. We define

$$
\operatorname{rinf}_{i \in \Lambda} \tilde{c}_{i}:=\left[\inf _{i \in \Lambda} c_{i}^{-}, \inf _{i \in \Lambda} c_{i}^{+}\right] \text {and } \operatorname{rsup}_{i \in \Lambda} \tilde{c}_{i}:=\left[\sup _{i \in \Lambda} c_{i}^{-}, \sup _{i \in \Lambda} c_{i}^{+}\right] .
$$

Let $M$ be a nonempty set. A function $f: M \rightarrow[[0,1]]$ is called an interval-valued fuzzy set (briefly, an IVF set) in $M$. Let $[[0,1]]^{M}$ stand for the set of all IVF sets in $M$. For every $f \in[[0,1]]^{M}$ and $\kappa_{1} \in M$, $f(x)=\left[f^{-}\left(\kappa_{1}\right), f^{+}\left(\kappa_{1}\right)\right]$ is called the degree of membership of an element $\kappa_{1}$ to $f$, where $f^{-}: M \rightarrow[0,1]$ and $f^{+}: M \rightarrow[0,1]$ are fuzzy sets in $M$ which are called a lower fuzzy set and an upper fuzzy set in $M$, respectively. For simplicity, we denote $f=\left[f^{-}, f^{+}\right]$. For every $f, g \in[[0,1]]^{M}$, we define

$$
f \subseteq g \Leftrightarrow f\left(\kappa_{1}\right) \preceq g\left(\kappa_{1}\right) \text { for all } \kappa_{1} \in M \text {, }
$$

and

$$
f=g \Leftrightarrow f\left(\kappa_{1}\right)=g\left(\kappa_{1}\right) \text { for all } \kappa_{1} \in M \text {. }
$$

Definition 2.1. [3] By a crossing cubic structure on a set $M$ we mean a pair $\left(M, \mathcal{C}_{(f, \xi)}\right)$ where

$$
\mathcal{C}_{(f, \xi)}:=\left\{\left\langle\kappa_{1}, f\left(\kappa_{1}\right), \xi\left(\kappa_{1}\right)\right\rangle \mid \kappa_{1} \in M\right\}
$$

in which $f$ is an interval-valued fuzzy set in $M$ and $\xi$ is an $\mathcal{N}$-function on $M$.

Definition 2.2. [3] A crossing cubic structure $\left(M, \mathcal{C}_{(f, \xi)}\right)$ on a $B C K / B C I$-algebra $M$ is called a crossing cubic subalgebra of $M$ if it satisfies:

$$
\left(\forall \kappa_{1}, \kappa_{2} \in M\right)\left(\begin{array}{l}
f\left(\kappa_{1} \rightsquigarrow \kappa_{2}\right) \succeq \operatorname{rmin}\left\{f\left(\kappa_{1}\right), f\left(\kappa_{2}\right)\right\} \\
\xi\left(\kappa_{1} \rightsquigarrow \kappa_{2}\right) \leq \max \left\{\xi\left(\kappa_{1}\right), \xi\left(\kappa_{2}\right)\right\}
\end{array}\right) .
$$

Proposition 2.3. Every crossing cubic subalgebra $\left(M, \mathcal{C}_{(f, \xi)}\right)$ of a $B C K / B C I$-algebra $M$ satisfies:

$$
\left(\forall \kappa_{1} \in M\right)\left(f(0) \succeq f\left(\kappa_{1}\right), \xi(0) \leq \xi\left(\kappa_{1}\right)\right) .
$$

\section{Crossing cubic ideals}

In what follows, let $M$ denote a BCK/BCI-algebra unless otherwise specified. 
Table 1: Cayley table for the binary operation " $\rightsquigarrow ”$

\begin{tabular}{c|cccc}
\hline$\rightsquigarrow$ & 0 & $\kappa_{1}$ & $\kappa_{2}$ & $\kappa_{3}$ \\
\hline 0 & 0 & 0 & 0 & $\kappa_{3}$ \\
$\kappa_{1}$ & $\kappa_{1}$ & 0 & $\kappa_{1}$ & $\kappa_{3}$ \\
$\kappa_{2}$ & $\kappa_{2}$ & $\kappa_{2}$ & 0 & $\kappa_{3}$ \\
$\kappa_{3}$ & $\kappa_{3}$ & $\kappa_{3}$ & $\kappa_{3}$ & 0 \\
\hline
\end{tabular}

Definition 3.1. A crossing cubic structure $\left(M, \mathcal{C}_{(f, \xi)}\right)$ on $M$ is called a crossing cubic ideal of $M$ if it satisfies:

$$
\left(\forall \kappa_{1}, \kappa_{2} \in M\right)\left(\begin{array}{l}
f(0) \succeq f\left(\kappa_{1}\right) \succeq \operatorname{rmin}\left\{f\left(\kappa_{1} \rightsquigarrow \kappa_{2}\right), f\left(\kappa_{2}\right)\right\} \\
\xi(0) \leq \xi\left(\kappa_{1}\right) \leq \max \left\{\xi\left(\kappa_{1} \rightsquigarrow \kappa_{2}\right), \xi\left(\kappa_{2}\right)\right\}
\end{array}\right) .
$$

Example 3.2. Let $M=\left\{0, \kappa_{1}, \kappa_{2}, \kappa_{3}\right\}$ be a set with the Cayley table which is given in Table 1 . Then $M$ is a BCI-algebra (see [2]). Let $\left(M, \mathcal{C}_{(f, \xi)}\right)$ be a crossing cubic structure on $M$ which is given by Table 2. It is routine to confirm that $\left(M, \mathcal{C}_{(f, \xi)}\right)$ is a crossing cubic ideal of $M$.

Table 2: Tabular representation of $\left(M, \mathcal{C}_{(f, \xi)}\right)$

\begin{tabular}{c|cc}
\hline$M$ & $f(x)$ & $\xi\left(\kappa_{1}\right)$ \\
\hline 0 & {$[0.43,0.83]$} & -0.82 \\
$\kappa_{1}$ & {$[0.35,0.76]$} & -0.56 \\
$\kappa_{2}$ & {$[0.33,0.53]$} & -0.73 \\
$\kappa_{3}$ & {$[0.15,0.47]$} & -0.32 \\
\hline
\end{tabular}

Proposition 3.3. Every crossing cubic ideal $\left(M, \mathcal{C}_{(f, \xi)}\right)$ of $M$ satisfies:

$\left(\forall \kappa_{1}, \kappa_{2} \in M\right)\left(\kappa_{1} \leq \kappa_{2} \Rightarrow f\left(\kappa_{1}\right) \succeq f\left(\kappa_{2}\right), \xi\left(\kappa_{1}\right) \leq \xi\left(\kappa_{2}\right)\right)$.

$\left(\forall \kappa_{1}, \kappa_{2}, \kappa_{3} \in M\right)\left(\kappa_{1} \rightsquigarrow \kappa_{2} \leq \kappa_{3} \Rightarrow f\left(\kappa_{1}\right) \succeq \operatorname{rmin}\left\{f\left(\kappa_{2}\right), f\left(\kappa_{3}\right)\right\}, \xi\left(\kappa_{1}\right) \leq \max \left\{\xi\left(\kappa_{2}\right), \xi\left(\kappa_{3}\right)\right\}\right)$.

$\left(\forall \kappa_{1}, \kappa_{2}, \kappa_{3} \in M\right)\left(f\left(\kappa_{1} \rightsquigarrow \kappa_{2}\right) \succeq \operatorname{rmin}\left\{f\left(\kappa_{1} \rightsquigarrow \kappa_{3}\right), f\left(\kappa_{3} \rightsquigarrow \kappa_{2}\right)\right\}, \xi\left(\kappa_{1} \rightsquigarrow \kappa_{2}\right) \leq \max \left\{\xi\left(\kappa_{1} \rightsquigarrow \kappa_{3}\right), \xi\left(\kappa_{3} \rightsquigarrow \kappa_{2}\right)\right\}\right)$.

$\left(\forall \kappa_{1}, \kappa_{2} \in M\right)\left(f\left(\kappa_{1} \rightsquigarrow \kappa_{2}\right)=f(0), \xi\left(\kappa_{1} \rightsquigarrow \kappa_{2}\right)=\xi(0) \Rightarrow f\left(\kappa_{1}\right) \succeq f\left(\kappa_{2}\right), \xi\left(\kappa_{1}\right) \leq \xi\left(\kappa_{2}\right)\right)$.

Proof. Let $\kappa_{1}, \kappa_{2} \in M$ be such that $\kappa_{1} \leq \kappa_{2}$. Then $\kappa_{1} \rightsquigarrow \kappa_{2}=0$, which implies from (17) that

$$
f\left(\kappa_{1}\right) \succeq \operatorname{rmin}\left\{f\left(\kappa_{1} \rightsquigarrow \kappa_{2}\right), f\left(\kappa_{2}\right)\right\}=\operatorname{rmin}\left\{f(0), f\left(\kappa_{2}\right)\right\}=f\left(\kappa_{2}\right),
$$

and $\xi\left(\kappa_{1}\right) \leq \max \left\{\xi\left(\kappa_{1} \rightsquigarrow \kappa_{2}\right), \xi\left(\kappa_{2}\right)\right\}=\max \left\{\xi(0), \xi\left(\kappa_{2}\right)\right\}=\xi\left(\kappa_{2}\right)$. Now, let $\kappa_{1}, \kappa_{2}, \kappa_{3} \in M$ be such that $\kappa_{1} \rightsquigarrow \kappa_{2} \leq \kappa_{3}$. Then $\left(\kappa_{1} \rightsquigarrow \kappa_{2}\right) \rightsquigarrow \kappa_{3}=0$, and so

$$
f\left(\kappa_{1} \rightsquigarrow \kappa_{2}\right) \succeq \operatorname{rmin}\left\{f\left(\left(\kappa_{1} \rightsquigarrow \kappa_{2}\right) \rightsquigarrow \kappa_{3}\right), f\left(\kappa_{3}\right)\right\}=\operatorname{rmin}\left\{f(0), f\left(\kappa_{3}\right)\right\}=f\left(\kappa_{3}\right),
$$

and

$$
\xi\left(\kappa_{1} \rightsquigarrow \kappa_{2}\right) \leq \max \left\{\xi\left(\left(\kappa_{1} \rightsquigarrow \kappa_{2}\right) \rightsquigarrow \kappa_{3}\right), \xi\left(\kappa_{3}\right)\right\}=\max \left\{\xi(0), \xi\left(\kappa_{3}\right)\right\}=\xi\left(\kappa_{3}\right) .
$$

It follows that

$$
f\left(\kappa_{1}\right) \succeq \operatorname{rmin}\left\{f\left(\kappa_{1} \rightsquigarrow \kappa_{2}\right), f\left(\kappa_{2}\right)\right\} \succeq \operatorname{rmin}\left\{f\left(\kappa_{3}\right), f\left(\kappa_{2}\right)\right\}
$$

and $\xi\left(\kappa_{1}\right) \leq \max \left\{\xi\left(\kappa_{1} \rightsquigarrow \kappa_{2}\right), \xi\left(\kappa_{2}\right)\right\} \leq \max \left\{\xi\left(\kappa_{3}\right), \xi\left(\kappa_{2}\right)\right\}$. Since $\left(\kappa_{1} \rightsquigarrow \kappa_{2}\right) \rightsquigarrow\left(\kappa_{1} \rightsquigarrow \kappa_{3}\right) \leq \kappa_{3} \rightsquigarrow \kappa_{2}$ for all $\kappa_{1}, \kappa_{2}, \kappa_{3} \in M,(20)$ is induced by $[19)$. Consider $\kappa_{1}, \kappa_{2} \in M$ satisfying $f\left(\kappa_{1} \rightsquigarrow \kappa_{2}\right)=f(0)$ and $\xi\left(\kappa_{1} \rightsquigarrow \kappa_{2}\right)=\xi(0)$. Then

$$
f\left(\kappa_{1}\right) \succeq \operatorname{rmin}\left\{f\left(\kappa_{1} \rightsquigarrow \kappa_{2}\right), f\left(\kappa_{2}\right)\right\}=\operatorname{rmin}\left\{f(0), f\left(\kappa_{2}\right)\right\}=f\left(\kappa_{2}\right),
$$

and $\xi\left(\kappa_{1}\right) \leq \max \left\{\xi\left(\kappa_{1} \rightsquigarrow \kappa_{2}\right), \xi\left(\kappa_{2}\right)\right\}=\max \left\{\xi(0), \xi\left(\kappa_{2}\right)\right\}=\xi\left(\kappa_{2}\right)$. This completes the proof. 
Proposition 3.4. Given a crossing cubic ideal $\left(M, \mathcal{C}_{(f, \xi)}\right)$ of $M$, the following are equivalent.

$$
\begin{aligned}
& \left(\forall \kappa_{1}, \kappa_{2} \in M\right)\left(\begin{array}{l}
f\left(\kappa_{1} \rightsquigarrow \kappa_{2}\right) \succeq f\left(\left(\kappa_{1} \rightsquigarrow \kappa_{2}\right) \rightsquigarrow \kappa_{2}\right) \\
\xi\left(\kappa_{1} \rightsquigarrow \kappa_{2}\right) \leq \xi\left(\left(\kappa_{1} \rightsquigarrow \kappa_{2}\right) \rightsquigarrow \kappa_{2}\right)
\end{array}\right) . \\
& \left(\forall \kappa_{1}, \kappa_{2}, \kappa_{3} \in M\right)\left(\begin{array}{l}
f\left(\left(\kappa_{1} \rightsquigarrow \kappa_{3}\right) \rightsquigarrow\left(\kappa_{2} \rightsquigarrow \kappa_{3}\right)\right) \succeq f\left(\left(\kappa_{1} \rightsquigarrow \kappa_{2}\right) \rightsquigarrow \kappa_{3}\right) \\
\xi\left(\left(\kappa_{1} \rightsquigarrow \kappa_{3}\right) \rightsquigarrow\left(\kappa_{2} \rightsquigarrow \kappa_{3}\right)\right) \leq \xi\left(\left(\kappa_{1} \rightsquigarrow \kappa_{2}\right) \rightsquigarrow \kappa_{3}\right)
\end{array}\right) .
\end{aligned}
$$

Proof. Suppose that (22) is valid and let $\kappa_{1}, \kappa_{2}, \kappa_{3} \in M$. Using (1), (7) and (8), we have

$$
\left(\left(\kappa_{1} \rightsquigarrow\left(\kappa_{2} \rightsquigarrow \kappa_{3}\right)\right) \rightsquigarrow \kappa_{3}\right) \rightsquigarrow \kappa_{3}=\left(\left(\kappa_{1} \rightsquigarrow \kappa_{3}\right) \rightsquigarrow\left(\kappa_{2} \rightsquigarrow \kappa_{3}\right)\right) \rightsquigarrow \kappa_{3} \leq\left(\kappa_{1} \rightsquigarrow \kappa_{2}\right) \rightsquigarrow \kappa_{3} .
$$

It follows from (18) that $f\left(\left(\left(\kappa_{1} \rightsquigarrow\left(\kappa_{2} \rightsquigarrow \kappa_{3}\right)\right) \rightsquigarrow \kappa_{3}\right) \rightsquigarrow \kappa_{3}\right) \succeq f\left(\left(\kappa_{1} \rightsquigarrow \kappa_{2}\right) \rightsquigarrow \kappa_{3}\right)$ and

$$
\left.\xi\left(\left(\kappa_{1} \rightsquigarrow\left(\kappa_{2} \rightsquigarrow \kappa_{3}\right)\right) \rightsquigarrow \kappa_{3}\right) \rightsquigarrow \kappa_{3}\right) \leq \xi\left(\left(\kappa_{1} \rightsquigarrow \kappa_{2}\right) \rightsquigarrow \kappa_{3}\right) .
$$

Combining these with (7) and (22) induces

$$
\begin{aligned}
f\left(\left(\kappa_{1} \rightsquigarrow \kappa_{3}\right) \rightsquigarrow\left(\kappa_{2} \rightsquigarrow \kappa_{3}\right)\right) & =f\left(\left(\kappa_{1} \rightsquigarrow\left(\kappa_{2} \rightsquigarrow \kappa_{3}\right)\right) \rightsquigarrow \kappa_{3}\right) \\
& \succeq f\left(\left(\left(\kappa_{1} \rightsquigarrow\left(\kappa_{2} \rightsquigarrow \kappa_{3}\right)\right) \rightsquigarrow \kappa_{3}\right) \rightsquigarrow \kappa_{3}\right) \\
& \succeq f\left(\left(\kappa_{1} \rightsquigarrow \kappa_{2}\right) \rightsquigarrow \kappa_{3}\right),
\end{aligned}
$$

and

$$
\begin{aligned}
\xi\left(\left(\kappa_{1} \rightsquigarrow \kappa_{3}\right) \rightsquigarrow\left(\kappa_{2} \rightsquigarrow \kappa_{3}\right)\right) & =\xi\left(\left(\kappa_{1} \rightsquigarrow\left(\kappa_{2} \rightsquigarrow \kappa_{3}\right)\right) \rightsquigarrow \kappa_{3}\right) \\
& \leq \xi\left(\left(\left(\kappa_{1} \rightsquigarrow\left(\kappa_{2} \rightsquigarrow \kappa_{3}\right)\right) \rightsquigarrow \kappa_{3}\right) \rightsquigarrow \kappa_{3}\right) \\
& \leq \xi\left(\left(\kappa_{1} \rightsquigarrow \kappa_{2}\right) \rightsquigarrow \kappa_{3}\right) .
\end{aligned}
$$

Conversely, assume that $\left(M, \mathcal{C}_{(f, \xi)}\right)$ satisfies $(23)$. Using $\kappa_{3}$ instead of $\kappa_{2}$ in 23 leads to

$$
f\left(\kappa_{1} \rightsquigarrow \kappa_{3}\right)=f\left(\left(\kappa_{1} \rightsquigarrow \kappa_{3}\right) \rightsquigarrow 0\right)=f\left(\left(\kappa_{1} \rightsquigarrow \kappa_{3}\right) \rightsquigarrow\left(\kappa_{3} \rightsquigarrow \kappa_{3}\right)\right) \succeq f\left(\left(\kappa_{1} \rightsquigarrow \kappa_{3}\right) \rightsquigarrow \kappa_{3}\right),
$$

and $\xi\left(\kappa_{1} \rightsquigarrow \kappa_{3}\right)=\xi\left(\left(\kappa_{1} \rightsquigarrow \kappa_{3}\right) \rightsquigarrow 0\right)=\xi\left(\left(\kappa_{1} \rightsquigarrow \kappa_{3}\right) \rightsquigarrow\left(\kappa_{3} \rightsquigarrow \kappa_{3}\right)\right) \leq \xi\left(\left(\kappa_{1} \rightsquigarrow \kappa_{3}\right) \rightsquigarrow \kappa_{3}\right)$ for all $\kappa_{1}, \kappa_{3} \in M$, by (3) and (6). This proves $(22)$.

We provide conditions for a crossing cubic structure $\left(M, \mathcal{C}_{(f, \xi)}\right)$ of $M$ to be a crossing cubic ideal of $M$.

Theorem 3.5. If a crossing cubic structure $\left(M, \mathcal{C}_{(f, \xi)}\right)$ of $M$ satisfies 19 and

$$
\left(\forall \kappa_{1} \in M\right)\left(f(0) \succeq f\left(\kappa_{1}\right), \xi(0) \leq \xi\left(\kappa_{1}\right)\right),
$$

then $\left(M, \mathcal{C}_{(f, \xi)}\right)$ is a crossing cubic ideal of $M$.

Proof. Let $\left(M, \mathcal{C}_{(f, \xi)}\right)$ be a crossing cubic structure of $M$ that satisfies $(19)$ and (24). Since $\kappa_{1} \rightsquigarrow\left(\kappa_{1} \rightsquigarrow\right.$ $\left.\kappa_{2}\right) \leq \kappa_{2}$ for all $\kappa_{1}, \kappa_{2} \in M$, it follows from 19 that $f\left(\kappa_{1}\right) \succeq \operatorname{rmin}\left\{f\left(\kappa_{1} \rightsquigarrow \kappa_{2}\right), f\left(\kappa_{2}\right)\right\}$ and $\xi\left(\kappa_{1}\right) \leq$ $\max \left\{\xi\left(\kappa_{1} \rightsquigarrow \kappa_{2}\right), \xi\left(\kappa_{2}\right)\right\}$. Therefore $\left(M, \mathcal{C}_{(f, \xi)}\right)$ is a crossing cubic ideal of $M$.

Given a crossing cubic structure $\left(M, \mathcal{C}_{(f, \xi)}\right)$ on $M$ and any element $v \in M$, we take the next set

$$
M_{v}:=\{x \in M \mid f(x) \succeq f(v), \xi(x) \leq \xi(v)\} .
$$

Theorem 3.6. If $\left(M, \mathcal{C}_{(f, \xi)}\right)$ is a crossing cubic ideal of $M$, then the set $M_{v}$ in 25) is an ideal of $M$ for all $v \in M$.

Proof. Let $v \in M$. It is clear that $0 \in M_{v}$. Let $\kappa_{1}, \kappa_{2} \in M$ be such that $\kappa_{1} \rightsquigarrow \kappa_{2} \in M_{v}$ and $\kappa_{2} \in M_{v}$. Then $f\left(\kappa_{1} \rightsquigarrow \kappa_{2}\right) \succeq f(v), \xi\left(\kappa_{1} \rightsquigarrow \kappa_{2}\right) \leq \xi(v), f\left(\kappa_{2}\right) \succeq f(v)$ and $\xi\left(\kappa_{2}\right) \leq \xi(v)$. It follows from (17) that

$$
f\left(\kappa_{1}\right) \succeq \operatorname{rmin}\left\{f\left(\kappa_{1} \rightsquigarrow \kappa_{2}\right), f\left(\kappa_{2}\right)\right\} \succeq f(v) \text { and } \xi\left(\kappa_{1}\right) \leq \max \left\{\xi\left(\kappa_{1} \rightsquigarrow \kappa_{2}\right), \xi\left(\kappa_{2}\right)\right\} \leq \xi(v) \text {. }
$$


Hence $\kappa_{1} \in M_{v}$ and therefore $M_{v}$ is an ideal of $M$.

Proposition 3.7. Let $\left(M, \mathcal{C}_{(f, \xi)}\right)$ be a crossing cubic structure on $M$. If $M_{v}$ is an ideal of $M$ for $v \in M$, then the next assertion is valid.

$$
\left(\forall \kappa_{1}, \kappa_{2}, \kappa_{3} \in M\right)\left(\begin{array}{l}
\operatorname{rmin}\left\{f\left(\kappa_{2} \rightsquigarrow \kappa_{3}\right), f\left(\kappa_{3}\right)\right\} \succeq f\left(\kappa_{1}\right) \Rightarrow f\left(\kappa_{2}\right) \succeq f\left(\kappa_{1}\right) \\
\max \left\{\xi\left(\kappa_{2} \rightsquigarrow \kappa_{3}\right), \xi\left(\kappa_{3}\right)\right\} \leq \xi\left(\kappa_{1}\right) \Rightarrow \xi\left(\kappa_{2}\right) \leq \xi\left(\kappa_{1}\right)
\end{array}\right) .
$$

Proof. Assume that $M_{v}$ is an ideal of $M$ for $v \in M$. Let $\kappa_{1}, \kappa_{2}, \kappa_{3} \in M$ be such that

$$
\operatorname{rmin}\left\{f\left(\kappa_{2} \rightsquigarrow \kappa_{3}\right), f\left(\kappa_{3}\right)\right\} \succeq f\left(\kappa_{1}\right) \text { and } \max \left\{\xi\left(\kappa_{2} \rightsquigarrow \kappa_{3}\right), \xi\left(\kappa_{3}\right)\right\} \leq \xi\left(\kappa_{1}\right) .
$$

Then $\kappa_{2} \rightsquigarrow \kappa_{3} \in M_{\kappa_{1}}$ and $\kappa_{3} \in M_{\kappa_{1}}$. Since $M_{\kappa_{1}}$ is an ideal of $M$, it follows that $\kappa_{2} \in M_{\kappa_{1}}$, that is, $f\left(\kappa_{2}\right) \succeq f\left(\kappa_{1}\right)$ and $\xi\left(\kappa_{2}\right) \leq \xi\left(\kappa_{1}\right)$.

Theorem 3.8. If a crossing cubic structure $\left(M, \mathcal{C}_{(f, \xi)}\right)$ on $M$ satisfies (24) and $(26)$, then the set $M_{v}$ in 25) is an ideal of $M$ for $v \in M$.

Proof. Let $v, \kappa_{1}, \kappa_{2} \in M$ be such that $\kappa_{1} \rightsquigarrow \kappa_{2} \in M_{v}$ and $\kappa_{2} \in M_{v}$. Then $f\left(\kappa_{1} \rightsquigarrow \kappa_{2}\right) \succeq f(v), \xi\left(\kappa_{1} \rightsquigarrow\right.$ $\left.\kappa_{2}\right) \leq \xi(v), f\left(\kappa_{2}\right) \succeq f(v)$ and $\xi\left(\kappa_{2}\right) \leq \xi(v)$ which imply that $\operatorname{rmin}\left\{f\left(\kappa_{1} \rightsquigarrow \kappa_{2}\right), f\left(\kappa_{2}\right)\right\} \succeq f(v)$ and $\max \left\{\xi\left(\kappa_{1} \rightsquigarrow \kappa_{2}\right), \xi\left(\kappa_{2}\right)\right\} \leq \xi(v)$. Combining these with 26 leads to $f\left(\kappa_{1}\right) \succeq f(v)$ and $\xi\left(\kappa_{1}\right) \leq \xi(v)$. Hence $\kappa_{1} \in M_{v}$. The condition (24) induces $0 \in M_{v}$. Therefore $M_{v}$ is an ideal of $M$.

We look at the relationship between a crossing cubic subalgebra and a crossing cubic ideal.

Theorem 3.9. In a BCK-algebra, every crossing cubic ideal is a crossing cubic subalgebra.

Proof. Let $\left(M, \mathcal{C}_{(f, \xi)}\right)$ be a crossing cubic ideal of a BCK-algebra $M$. Using (3), (5), (7) and (17), we get

$$
\begin{aligned}
f\left(\kappa_{1} \rightsquigarrow \kappa_{2}\right) & \succeq \operatorname{rmin}\left\{f\left(\left(\kappa_{1} \rightsquigarrow \kappa_{2}\right) \rightsquigarrow \kappa_{1}\right), f\left(\kappa_{1}\right)\right\} \\
& =\operatorname{rmin}\left\{f\left(\left(\kappa_{1} \rightsquigarrow \kappa_{1}\right) \rightsquigarrow \kappa_{2}\right), f\left(\kappa_{1}\right)\right\} \\
& =\operatorname{rmin}\left\{f(0), f\left(\kappa_{1}\right)\right\} \\
& \succeq \operatorname{rmin}\left\{f\left(\kappa_{1}\right), f\left(\kappa_{2}\right)\right\}
\end{aligned}
$$

and

$$
\begin{aligned}
\xi\left(\kappa_{1} \rightsquigarrow \kappa_{2}\right) & \leq \max \left\{\xi\left(\left(\kappa_{1} \rightsquigarrow \kappa_{2}\right) \rightsquigarrow \kappa_{1}\right), \xi\left(\kappa_{1}\right)\right\} \\
& =\max \left\{\xi\left(\left(\kappa_{1} \rightsquigarrow \kappa_{1}\right) \rightsquigarrow \kappa_{2}\right), \xi\left(\kappa_{1}\right)\right\} \\
& =\max \left\{\xi(0), \xi\left(\kappa_{1}\right)\right\} \\
& \leq \max \left\{\xi\left(\kappa_{1}\right), \xi\left(\kappa_{2}\right)\right\}
\end{aligned}
$$

for all $\kappa_{1}, \kappa_{2} \in M$. Hence $\left(M, \mathcal{C}_{(f, \xi)}\right)$ is a crossing cubic subalgebra of $M$.

The following example shows that any crossing cubic subalgebra may not be a crossing cubic ideal.

Example 3.10. Let $M:=\left\{0, \kappa_{1}, \kappa_{2}, \kappa_{3}, \kappa_{4}\right\}$ be a set with the Cayley table in Table 3 . Then $M$ is a BCK-

Table 3: Cayley table for the binary operation "»”"

\begin{tabular}{c|ccccc}
\hline$\rightsquigarrow$ & 0 & $\kappa_{1}$ & $\kappa_{2}$ & $\kappa_{3}$ & $\kappa_{4}$ \\
\hline 0 & 0 & 0 & 0 & 0 & 0 \\
$\kappa_{1}$ & $\kappa_{1}$ & 0 & 0 & 0 & 0 \\
$\kappa_{2}$ & $\kappa_{2}$ & $\kappa_{1}$ & 0 & $\kappa_{1}$ & 0 \\
$\kappa_{3}$ & $\kappa_{3}$ & $\kappa_{3}$ & $\kappa_{3}$ & 0 & 0 \\
$\kappa_{4}$ & $\kappa_{4}$ & $\kappa_{4}$ & $\kappa_{4}$ & $\kappa_{3}$ & 0 \\
\hline
\end{tabular}

algebra (see [6]). Let $\left(M, \mathcal{C}_{(f, \xi)}\right)$ be a crossing cubic structure on $M$ which is given by Table 4. It is routine to confirm that $\left(M, \mathcal{C}_{(f, \xi)}\right)$ is a crossing cubic subalgebra of $M$. But it is not a crossing cubic ideal of $M$ since 
Table 4: Tabular representation of $\left(M, \mathcal{C}_{(f, \xi)}\right)$

\begin{tabular}{c|cc}
\hline$M$ & $f(x)$ & $\xi\left(\kappa_{1}\right)$ \\
\hline 0 & {$[0.39,0.76]$} & -0.73 \\
$\kappa_{1}$ & {$[0.35,0.67]$} & -0.73 \\
$\kappa_{2}$ & {$[0.39,0.76]$} & -0.26 \\
$\kappa_{3}$ & {$[0.25,0.58]$} & -0.62 \\
$\kappa_{4}$ & {$[0.15,0.47]$} & -0.47 \\
\hline
\end{tabular}

$$
\begin{gathered}
\xi\left(\kappa_{2}\right)=-0.26 \not-0.62=\max \left\{\xi\left(\kappa_{2} \rightsquigarrow \kappa_{3}\right), \xi\left(\kappa_{3}\right)\right\} \text { and } / \text { or } \\
f\left(\kappa_{4}\right)=[0.15,0.47] \nsucceq[0.25,0.58]=\operatorname{rmin}\left\{f\left(\kappa_{4} \rightsquigarrow \kappa_{3}\right), f\left(\kappa_{3}\right)\right\} .
\end{gathered}
$$

We know that any crossing cubic ideal is not a crossing cubic subalgebra in a BCI-algebra as seen in the next example.

Example 3.11. Let $(\mathbb{Z},-, 0)$ be the adjoint BCI-algebra of the additive group $(\mathbb{Z},+, 0)$ of integers and $(K, *, 0)$ be a BCI-algebra. Then the direct product $M:=K \times \mathbb{Z}$ of $K$ and $\mathbb{Z}$ is a BCI-algebra (see [2]). Define a crossing cubic structure $\left(M, \mathcal{C}_{(f, \xi)}\right)$ on $M$ as follows:

$$
\begin{aligned}
& f: M \rightarrow[[0,1]], \kappa_{1} \mapsto \begin{cases}\tilde{\alpha} & \text { if } x \in K \times(\mathbb{N} \cup\{0\}), \\
{[0,0]} & \text { otherwise }\end{cases} \\
& \xi: M \rightarrow[-1,0], \kappa_{1} \mapsto \begin{cases}k & \text { if } x \in K \times(\mathbb{N} \cup\{0\}), \\
0 & \text { otherwise }\end{cases}
\end{aligned}
$$

where $\mathbb{N}$ is the set of all natural numbers, $k \in[-1,0)$ and $\tilde{\alpha}$ is an interval number which is not $[0,0]$. By routine calculations, we know that $\left(M, \mathcal{C}_{(f, \xi)}\right)$ is a crossing cubic ideal of $M$. If we take $x:=(0,0)$ and $y:=(0,1)$, then $z:=x \rightsquigarrow y=(0,0) \rightsquigarrow(0,1)=(0,-1)$. Hence $\xi(x \rightsquigarrow y)=\xi(z)=0 \not \leq k=\max \{\xi(x), \xi(y)\}$ and/or $f(x \rightsquigarrow y)=f(z)=[0,0] \nsucceq \tilde{\alpha}=\operatorname{rmin}\{f(x), f(y)\}$. Therefore $\left(M, \mathcal{C}_{(f, \xi)}\right)$ is not a crossing cubic subalgebra of $M$.

Based on Example 3.11, we can define the following:

Definition 3.12. Let $M$ be a BCI-algebra. A crossing cubic ideal $\left(M, \mathcal{C}_{(f, \xi)}\right)$ of $M$ is said to be closed if it is also a crossing cubic subalgebra of $M$.

Example 3.13. Let $M:=\left\{0, \kappa_{1}, \kappa_{2}, \kappa_{3}, \kappa_{4}\right\}$ be a set with the Cayley table in Table 5. Then $M$ is a BCI-

Table 5: Cayley table for the binary operation " $\rightsquigarrow$ ”

\begin{tabular}{c|ccccc}
\hline$\rightsquigarrow$ & 0 & $\kappa_{1}$ & $\kappa_{2}$ & $\kappa_{3}$ & $\kappa_{4}$ \\
\hline 0 & 0 & 0 & $\kappa_{2}$ & $\kappa_{3}$ & $\kappa_{4}$ \\
$\kappa_{1}$ & $\kappa_{1}$ & 0 & $\kappa_{2}$ & $\kappa_{3}$ & $\kappa_{4}$ \\
$\kappa_{2}$ & $\kappa_{2}$ & $\kappa_{2}$ & 0 & $\kappa_{4}$ & $\kappa_{3}$ \\
$\kappa_{3}$ & $\kappa_{3}$ & $\kappa_{3}$ & $\kappa_{4}$ & 0 & $\kappa_{2}$ \\
$\kappa_{4}$ & $\kappa_{4}$ & $\kappa_{4}$ & $\kappa_{3}$ & $\kappa_{2}$ & 0 \\
\hline
\end{tabular}

algebra (see [2]). Let $\left(M, \mathcal{C}_{(f, \xi)}\right)$ be a crossing cubic structure on $M$ which is given by Table 6 . It is routine to check that $\left(M, \mathcal{C}_{(f, \xi)}\right)$ is a closed crossing cubic ideal of $M$.

We provide conditions for a crossing cubic structure to be a closed crossing cubic ideal. 
Table 6: Tabular representation of $\left(M, \mathcal{C}_{(f, \xi)}\right)$

\begin{tabular}{c|cc}
\hline$M$ & $f(x)$ & $\xi\left(\kappa_{1}\right)$ \\
\hline 0 & {$[0.5,0.9]$} & -0.8 \\
$\kappa_{1}$ & {$[0.4,0.8]$} & -0.6 \\
$\kappa_{2}$ & {$[0.2,0.6]$} & -0.3 \\
$\kappa_{3}$ & {$[0.2,0.6]$} & -0.5 \\
$\kappa_{4}$ & {$[0.3,0.7]$} & -0.3 \\
\hline
\end{tabular}

Theorem 3.14. Let $\left(M, \mathcal{C}_{(f, \xi)}\right)$ be a crossing cubic structure on a BCI-algebra $M$ in which

$$
\begin{aligned}
& f: M \rightarrow[[0,1]], \kappa_{1} \mapsto\left\{\begin{array}{cc}
\tilde{\alpha} & \text { if } x \in M_{+}, \\
\tilde{\beta} & \text { otherwise, }
\end{array}\right. \\
& \xi: M \rightarrow[-1,0], \kappa_{1} \mapsto \begin{cases}t_{1} & \text { if } x \in M_{+}, \\
t_{2} & \text { otherwise }\end{cases}
\end{aligned}
$$

where $M_{+}=\{x \in M \mid 0 \leq x\}$, $\tilde{\alpha}$ and $\tilde{\beta}$ are interval numbers with $\tilde{\alpha} \succ \tilde{\beta}$, and $t_{1}, t_{2} \in[-1,0]$ with $t_{1}<t_{2}$. Then $\left(M, \mathcal{C}_{(f, \xi)}\right)$ is a closed crossing cubic ideal of $M$.

Proof. Since $0 \in M_{+}$, we have $f(0)=\tilde{\alpha} \succeq f\left(\kappa_{1}\right)$ and $\xi(0)=t_{1} \leq \xi\left(\kappa_{1}\right)$ for all $\kappa_{1} \in M$. Let $\kappa_{1}, \kappa_{2} \in M$. If $\kappa_{1} \in M_{+}$, then $f\left(\kappa_{1}\right)=\tilde{\alpha} \succeq \operatorname{rmin}\left\{f\left(\kappa_{1} \rightsquigarrow \kappa_{2}\right), f\left(\kappa_{2}\right)\right\}$, and $\xi\left(\kappa_{1}\right)=t_{1} \leq \max \left\{\xi\left(\kappa_{1} \rightsquigarrow \kappa_{2}\right), \xi\left(\kappa_{2}\right)\right\}$. Suppose that $\kappa_{1} \notin M$. If $\kappa_{2} \in M_{+}$, then $\kappa_{1} \rightsquigarrow \kappa_{2} \notin M_{+}$, and if $\kappa_{1} \rightsquigarrow \kappa_{2} \in M_{+}$, then $\kappa_{2} \notin M_{+}$. In either case, we have $f\left(\kappa_{1}\right)=\tilde{\beta}=\operatorname{rmin}\left\{f\left(\kappa_{1} \rightsquigarrow \kappa_{2}\right), f\left(\kappa_{2}\right)\right\}$ and $\xi\left(\kappa_{1}\right)=t_{2}=\max \left\{\xi\left(\kappa_{1} \rightsquigarrow \kappa_{2}\right), \xi\left(\kappa_{2}\right)\right\}$. Hence $\left(M, \mathcal{C}_{(f, \xi)}\right)$ is a crossing cubic ideal of $M$. For every $\kappa_{1}, \kappa_{2} \in M$, if any one of them does not belong to $M_{+}$, then $f\left(\kappa_{1} \rightsquigarrow \kappa_{2}\right) \succeq \tilde{\beta}=\operatorname{rmin}\left\{f\left(\kappa_{1}\right), f\left(\kappa_{2}\right)\right\}$, and $\xi\left(\kappa_{1} \rightsquigarrow \kappa_{2}\right) \leq t_{2}=\max \left\{\xi\left(\kappa_{1}\right), \xi\left(\kappa_{2}\right)\right\}$. If $\kappa_{1}, \kappa_{2} \in M_{+}$, then $\kappa_{1} \rightsquigarrow \kappa_{2} \in M_{+}$and thus $f\left(\kappa_{1} \rightsquigarrow \kappa_{2}\right)=\tilde{\alpha}=\operatorname{rmin}\left\{f\left(\kappa_{1}\right), f\left(\kappa_{2}\right)\right\}$, and $\xi\left(\kappa_{1} \rightsquigarrow \kappa_{2}\right)=t_{1}=\max \left\{\xi\left(\kappa_{1}\right), \xi\left(\kappa_{2}\right)\right\}$. Thus $\left(M, \mathcal{C}_{(f, \xi)}\right)$ is a crossing cubic subalgebra of $M$. Consequently, $\left(M, \mathcal{C}_{(f, \xi)}\right)$ is a closed crossing cubic ideal of $M$.

Definition 3.15. Let $M$ be a BCI-algebra. If a crossing cubic structure $\left(M, \mathcal{C}_{(f, \xi)}\right)$ on $M$ satisfies:

$$
\left(\forall \kappa_{1} \in M\right)\left(f\left(0 \rightsquigarrow \kappa_{1}\right) \succeq f\left(\kappa_{1}\right), \xi\left(0 \rightsquigarrow \kappa_{1}\right) \leq \xi\left(\kappa_{1}\right)\right),
$$

then we say that $\left(M, \mathcal{C}_{(f, \xi)}\right)$ is a 0 -crossing cubic structure on $M$.

Theorem 3.16. In a BCI-algebra, every crossing cubic subalgebra is a 0-crossing cubic structure.

Proof. Let $\left(M, \mathcal{C}_{(f, \xi)}\right)$ be a crossing cubic subalgebra of a BCI-algebra $M$. Using (15) and (16), we get

$$
f\left(0 \rightsquigarrow \kappa_{1}\right) \succeq \operatorname{rmin}\left\{f(0), f\left(\kappa_{1}\right)\right\} \succeq \operatorname{rmin}\left\{f\left(\kappa_{1}\right), f\left(\kappa_{1}\right)\right\}=f\left(\kappa_{1}\right),
$$

and

$$
\xi\left(0 \rightsquigarrow \kappa_{1}\right) \leq \max \left\{\xi(0), \xi\left(\kappa_{1}\right)\right\} \leq \max \left\{\xi\left(\kappa_{1}\right), \xi\left(\kappa_{1}\right)\right\}=\xi\left(\kappa_{1}\right),
$$

for all $\kappa_{1} \in M$. Therefore $\left(M, \mathcal{C}_{(f, \xi)}\right)$ is a 0 -crossing cubic structure on $M$.

Corollary 3.17. In a BCI-algebra, every closed crossing cubic ideal is a 0-crossing cubic structure.

We explore the conditions under which crossing cubic ideal is closed.

Theorem 3.18. Let $\left(M, \mathcal{C}_{(f, \xi)}\right)$ be a crossing cubic ideal of a BCI-algebra $M$. If it is a 0 -crossing cubic structure on $M$, then $\left(M, \mathcal{C}_{(f, \xi)}\right)$ is a crossing cubic subalgebra, and hence a closed crossing cubic ideal of $M$. 
Proof. Since $\left(\kappa_{1} \rightsquigarrow \kappa_{2}\right) \rightsquigarrow \kappa_{1} \leq 0 \rightsquigarrow \kappa_{2}$ for all $\kappa_{1}, \kappa_{2} \in M$, it follows from (19) and (27) that

$$
f\left(\kappa_{1} \rightsquigarrow \kappa_{2}\right) \succeq \operatorname{rmin}\left\{f\left(\kappa_{1}\right), f\left(0 \rightsquigarrow \kappa_{2}\right)\right\} \succeq \operatorname{rmin}\left\{f\left(\kappa_{1}\right), f\left(\kappa_{2}\right)\right\},
$$

and

$$
\xi\left(\kappa_{1} \rightsquigarrow \kappa_{2}\right) \leq \max \left\{\xi\left(\kappa_{1}\right), \xi\left(0 \rightsquigarrow \kappa_{2}\right)\right\} \leq \max \left\{\xi\left(\kappa_{1}\right), \xi\left(\kappa_{2}\right)\right\} .
$$

Therefore $\left(M, \mathcal{C}_{(f, \xi)}\right)$ is a crossing cubic subalgebra of $M$, which completes the proof.

Let $M$ be a BCI-algebra. For every $\kappa_{1} \in M$ and a natural number $n$, we define $\kappa_{1}^{n}$ as follows:

$$
\kappa_{1}^{1}=\kappa_{1} \text { and } \kappa_{1}^{n+1}=\kappa_{1} \rightsquigarrow\left(0 \rightsquigarrow \kappa_{1}^{n}\right) .
$$

The element $\kappa_{1}$ of a BCI-algebra $M$ is called having a finite period (see [7]) if $\kappa_{1}^{n} \in M_{+}$for some natural number $n$. We represent the period of $\kappa_{1}$ by $\left|\kappa_{1}\right|$, and it is given as follows:

$$
\left|\kappa_{1}\right|=\min \left\{n \in \mathbb{N} \mid \kappa_{1}^{n} \in M_{+}\right\} .
$$

Theorem 3.19. If $M$ is a BCI-algebra in which every element has finite period, then every crossing cubic ideal of $M$ is a crossing cubic subalgebra, and hence a closed crossing cubic ideal of $M$.

Proof. Let $\left(M, \mathcal{C}_{(f, \xi)}\right)$ be a crossing cubic ideal of $M$ and let $\kappa_{1}$ be an element of $M$ with $\left|\kappa_{1}\right|=n$. Then

$$
\begin{aligned}
\left(0 \rightsquigarrow \kappa_{1}^{n-1}\right) \rightsquigarrow \kappa_{1} & =\left(0 \rightsquigarrow\left(0 \rightsquigarrow\left(0 \rightsquigarrow \kappa_{1}^{n-1}\right)\right)\right) \rightsquigarrow \kappa_{1} \\
& =\left(0 \rightsquigarrow \kappa_{1}\right) \rightsquigarrow\left(0 \rightsquigarrow\left(0 \rightsquigarrow \kappa_{1}^{n-1}\right)\right) \\
& =0 \rightsquigarrow\left(\kappa_{1} \rightsquigarrow\left(0 \rightsquigarrow \kappa_{1}^{n-1}\right)\right)=0 \rightsquigarrow \kappa_{1}^{n}=0,
\end{aligned}
$$

which implies that $f\left(\left(0 \rightsquigarrow \kappa_{1}^{n-1}\right) \rightsquigarrow \kappa_{1}\right)=f(0) \succeq f\left(\kappa_{1}\right)$ and $\xi\left(\left(0 \rightsquigarrow \kappa_{1}^{n-1}\right) \rightsquigarrow \kappa_{1}\right)=\xi(0) \leq \xi\left(\kappa_{1}\right)$. It follows from (17) that

$$
\begin{aligned}
& f\left(0 \rightsquigarrow \kappa_{1}^{n-1}\right) \succeq \operatorname{rmin}\left\{f\left(\left(0 \rightsquigarrow \kappa_{1}^{n-1}\right) \rightsquigarrow \kappa_{1}\right), f\left(\kappa_{1}\right)\right\} \succeq f\left(\kappa_{1}\right), \\
& \xi\left(0 \rightsquigarrow \kappa_{1}^{n-1}\right) \leq \max \left\{\xi\left(\left(0 \rightsquigarrow \kappa_{1}^{n-1}\right) \rightsquigarrow \kappa_{1}\right), \xi\left(\kappa_{1}\right)\right\} \leq \xi\left(\kappa_{1}\right) .
\end{aligned}
$$

Also note that

$$
\begin{aligned}
\left(0 \rightsquigarrow \kappa_{1}^{n-2}\right) \rightsquigarrow \kappa_{1} & =\left(0 \rightsquigarrow\left(0 \rightsquigarrow\left(0 \rightsquigarrow \kappa_{1}^{n-2}\right)\right)\right) \rightsquigarrow \kappa_{1} \\
& =\left(0 \rightsquigarrow \kappa_{1}\right) \rightsquigarrow\left(0 \rightsquigarrow\left(0 \rightsquigarrow \kappa_{1}^{n-2}\right)\right) \\
& =0 \rightsquigarrow\left(\kappa_{1} \rightsquigarrow\left(0 \rightsquigarrow \kappa_{1}^{n-2}\right)\right)=0 \rightsquigarrow \kappa_{1}^{n-1} .
\end{aligned}
$$

Using $(28)$ leads to

$$
f\left(\left(0 \rightsquigarrow \kappa_{1}^{n-2}\right) \rightsquigarrow \kappa_{1}\right)=f\left(0 \rightsquigarrow \kappa_{1}^{n-1}\right) \succeq f\left(\kappa_{1}\right),
$$

and

$$
\xi\left(\left(0 \rightsquigarrow \kappa_{1}^{n-2}\right) \rightsquigarrow \kappa_{1}\right)=\xi\left(0 \rightsquigarrow \kappa_{1}^{n-1}\right) \leq \xi\left(\kappa_{1}\right) .
$$

It follows from 17 that $f\left(0 \rightsquigarrow \kappa_{1}^{n-2}\right) \succeq \operatorname{rmin}\left\{f\left(\left(0 \rightsquigarrow \kappa_{1}^{n-2}\right) \rightsquigarrow \kappa_{1}\right), f\left(\kappa_{1}\right)\right\} \succeq f\left(\kappa_{1}\right)$ and

$$
\xi\left(0 \rightsquigarrow \kappa_{1}^{n-2}\right) \leq \max \left\{\xi\left(\left(0 \rightsquigarrow \kappa_{1}^{n-2}\right) \rightsquigarrow \kappa_{1}\right), \xi\left(\kappa_{1}\right)\right\} \leq \xi\left(\kappa_{1}\right) .
$$

If this process repeats, it will lead to $f\left(0 \rightsquigarrow \kappa_{1}\right) \succeq f\left(\kappa_{1}\right)$ and $\xi\left(0 \rightsquigarrow \kappa_{1}\right) \leq \xi\left(\kappa_{1}\right)$. So, by Theorem 3.18 $\left(M, \mathcal{C}_{(f, \xi)}\right)$ is a crossing cubic subalgebra, and therefore a closed crossing cubic ideal of $M$.

Definition 3.20. Let $M$ be a BCK-algebra with the condition $(S)$. A crossing cubic structure $\left(M, \mathcal{C}_{(f, \xi)}\right)$ on $M$ is called a crossing cubic o-subalgebra of $M$ if it satisfies:

$$
\left(\forall \kappa_{1}, \kappa_{2} \in M\right)\left(\begin{array}{l}
f\left(\kappa_{1} \circ \kappa_{2}\right) \succeq \operatorname{rmin}\left\{f\left(\kappa_{1}\right), f\left(\kappa_{2}\right)\right\} \\
\xi\left(\kappa_{1} \circ \kappa_{2}\right) \leq \max \left\{\xi\left(\kappa_{1}\right), \xi\left(\kappa_{2}\right)\right\}
\end{array}\right) .
$$


Theorem 3.21. In a BCK-algebra with the condition (S), every crossing cubic ideal is a crossing cubic o-subalgebra.

Proof. Let $\left(M, \mathcal{C}_{(f, \xi)}\right)$ be a crossing cubic ideal of $M$. Since $\left(\kappa_{1} \circ \kappa_{2}\right) \rightsquigarrow \kappa_{1} \leq \kappa_{2}$ for all $\kappa_{1}, \kappa_{2} \in M$, it follows from (19) that $f\left(\kappa_{1} \circ \kappa_{2}\right) \succeq \operatorname{rmin}\left\{f\left(\kappa_{1}\right), f\left(\kappa_{2}\right)\right\}$ and $\xi\left(\kappa_{1} \circ \kappa_{2}\right) \leq \max \left\{\xi\left(\kappa_{1}\right), \xi\left(\kappa_{2}\right)\right\}$ for all $\kappa_{1}, \kappa_{2} \in M$. Therefore $\left(M, \mathcal{C}_{(f, \xi)}\right)$ is a crossing cubic o-subalgebra of $M$.

Theorem 3.22. Given a crossing cubic structure $\left(M, \mathcal{C}_{(f, \xi)}\right)$ on a BCK-algebra $M$ with the condition (S), the following assertions are equivalent.

(i) $\left(M, \mathcal{C}_{(f, \xi)}\right)$ is a crossing cubic ideal of $M$.

(ii) $\left(M, \mathcal{C}_{(f, \xi)}\right)$ satisfies:

$$
\left(\forall \kappa_{1}, \kappa_{2}, \kappa_{3} \in M\right)\left(\kappa_{1} \leq \kappa_{2} \circ \kappa_{3} \Rightarrow f\left(\kappa_{1}\right) \succeq \operatorname{rmin}\left\{f\left(\kappa_{2}\right), f\left(\kappa_{3}\right)\right\}, \xi\left(\kappa_{1}\right) \leq \max \left\{\xi\left(\kappa_{2}\right), \xi\left(\kappa_{3}\right)\right\}\right) .
$$

Proof. Suppose that $\left(M, \mathcal{C}_{(f, \xi)}\right)$ is a crossing cubic ideal of $M$ and let $\kappa_{1}, \kappa_{2}, \kappa_{3} \in M$ be such that $\kappa_{1} \leq \kappa_{2} \circ \kappa_{3}$. Then $\kappa_{1} \rightsquigarrow\left(\kappa_{2} \circ \kappa_{3}\right)=0$, and so

$$
f\left(\kappa_{1}\right) \succeq \operatorname{rmin}\left\{f\left(\kappa_{1} \rightsquigarrow\left(\kappa_{2} \circ \kappa_{3}\right)\right), f\left(\kappa_{2} \circ \kappa_{3}\right)\right\} \succeq \operatorname{rmin}\left\{f(0), f\left(\kappa_{2} \circ \kappa_{3}\right)\right\}=f\left(\kappa_{2} \circ \kappa_{3}\right) \succeq \operatorname{rmin}\left\{f\left(\kappa_{2}\right), f\left(\kappa_{3}\right)\right\},
$$

and

$$
\xi\left(\kappa_{1}\right) \leq \max \left\{\xi\left(\kappa_{1} \rightsquigarrow\left(\kappa_{2} \circ \kappa_{3}\right)\right), \xi\left(\kappa_{2} \circ \kappa_{3}\right)\right\} \leq \max \left\{\xi(0), \xi\left(\kappa_{2} \circ \kappa_{3}\right)\right\}=\xi\left(\kappa_{2} \circ \kappa_{3}\right) \leq \max \left\{\xi\left(\kappa_{2}\right), \xi\left(\kappa_{3}\right)\right\},
$$

by (17) and Theorem 3.21 .

Conversely, assume that $\left(M, \mathcal{C}_{(f, \xi)}\right)$ satisfies 30 . Since $0 \leq \kappa_{1} \circ \kappa_{1}$ for all $\kappa_{1} \in M$, we have

$$
f(0) \succeq \operatorname{rmin}\left\{f\left(\kappa_{1}\right), f\left(\kappa_{1}\right)\right\}=f\left(\kappa_{1}\right) \text { and } \xi(0) \leq \max \left\{\xi\left(\kappa_{1}\right), \xi\left(\kappa_{1}\right)\right\}=\xi\left(\kappa_{1}\right),
$$

for all $\kappa_{1} \in M$ by (30). Note that $\kappa_{1} \leq\left(\kappa_{1} \rightsquigarrow \kappa_{2}\right) \circ \kappa_{2}$ for all $\kappa_{1}, \kappa_{2} \in M$. Using (30) leads to

$$
f\left(\kappa_{1}\right) \succeq \operatorname{rmin}\left\{f\left(\kappa_{1} \rightsquigarrow \kappa_{2}\right), f\left(\kappa_{2}\right)\right\} \text { and } \xi\left(\kappa_{1}\right) \leq \max \left\{\xi\left(\kappa_{1} \rightsquigarrow \kappa_{2}\right), \xi\left(\kappa_{2}\right)\right\},
$$

for all $\kappa_{1}, \kappa_{2} \in M$. Hence $\left(M, \mathcal{C}_{(f, \xi)}\right)$ is a crossing cubic ideal of $M$.

\section{Translations of crossing cubic subalgebras and crossing cubic ideals}

Given a crossing cubic structure $\left(M, \mathcal{C}_{(f, \xi)}\right)$ on a set $M$, we denote

$$
\mathrm{T}:=1-\sup \left\{f^{+}\left(\kappa_{1}\right) \mid \kappa_{1} \in M\right\} \text { and } \perp:=-1-\inf \left\{\xi\left(\kappa_{1}\right) \mid \kappa_{1} \in M\right\} .
$$

For every $\tilde{\alpha}=\left[\alpha^{-}, \alpha^{+}\right] \in[[0,1]]$ with $\alpha^{+} \leq \mathrm{T}$ and $k \in[-1,0]$ with $k \geq \perp$, we define

$$
\begin{aligned}
& f_{\tilde{\alpha}}^{T}: M \rightarrow[[0,1]], \kappa_{1} \mapsto f\left(\kappa_{1}\right)+\tilde{\alpha}, \\
& \xi_{k}^{T}: M \rightarrow[-1,0], \kappa_{1} \mapsto \xi\left(\kappa_{1}\right)+k .
\end{aligned}
$$

Then $\left(M, \mathcal{C}_{\left(f_{\tilde{\alpha}}^{T}, \xi_{k}^{T}\right)}\right)$ is a crossing cubic structure on $M$, which is called the $(\tilde{\alpha}, k)$-translation of $\left(M, \mathcal{C}_{(f, \xi)}\right)$.

Example 4.1. Let $\left(M, \mathcal{C}_{(f, \xi)}\right)$ be a crossing cubic structure on a set $M:=\left\{0, \kappa_{1}, \kappa_{2}, \kappa_{3}, \kappa_{4}\right\}$ which is given by Table 7 .

Then $\mathrm{T}=1-\sup \left\{f^{+}\left(\kappa_{1}\right) \mid \kappa_{1} \in M\right\}=0.19$ and $\perp:=-1-\inf \left\{\xi\left(\kappa_{1}\right) \mid \kappa_{1} \in M\right\}=-0.36$. If we take $\tilde{\alpha}=[0.07,0.13]$ and $k=-0.32$, then the $(\tilde{\alpha}, k)$-translation $\left(M, \mathcal{C}_{\left(f_{\tilde{\alpha}}^{T}, \xi_{k}^{T}\right)}\right)$ of $\left(M, \mathcal{C}_{(f, \xi)}\right)$ is given by Table 8 
Table 7: Tabular representation of $\left(M, \mathcal{C}_{(f, \xi)}\right)$

\begin{tabular}{c|cc}
\hline$M$ & $f(x)$ & $\xi\left(\kappa_{1}\right)$ \\
\hline 0 & {$[0.53,0.73]$} & -0.23 \\
$\kappa_{1}$ & {$[0.37,0.81]$} & -0.64 \\
$\kappa_{2}$ & {$[0.28,0.62]$} & -0.33 \\
$\kappa_{3}$ & {$[0.24,0.56]$} & -0.57 \\
$\kappa_{4}$ & {$[0.33,0.68]$} & -0.38 \\
\hline
\end{tabular}

Table 8: Tabular representation of $\left(M, \mathcal{C}_{\left(f_{\tilde{\alpha}}^{T}, \xi_{k}^{T}\right)}\right)$

\begin{tabular}{c|cc}
\hline$M$ & $f(x)$ & $\xi\left(\kappa_{1}\right)$ \\
\hline 0 & {$[0.60,0.86]$} & -0.55 \\
$\kappa_{1}$ & {$[0.44,0.94]$} & -0.96 \\
$\kappa_{2}$ & {$[0.35,0.75]$} & -0.65 \\
$\kappa_{3}$ & {$[0.31,0.69]$} & -0.89 \\
$\kappa_{4}$ & {$[0.40,0.81]$} & -0.70 \\
\hline
\end{tabular}

Theorem 4.2. If $\left(M, \mathcal{C}_{(f, \xi)}\right)$ is a crossing cubic subalgebra or a crossing cubic ideal of $M$, then its $(\tilde{\alpha}, k)$ translation $\left(M, \mathcal{C}_{\left(f_{\tilde{\alpha}}^{T}, \xi_{k}^{T}\right)}\right)$ is also a crossing cubic subalgebra or a crossing cubic ideal of $M$ for all $\tilde{\alpha}=$ $\left[\alpha^{-}, \alpha^{+}\right] \in[[0,1]]$ and $k \in[-1,0]$ with $\alpha^{+} \leq \mathrm{T}$ and $k \geq \perp$, respectively.

Proof. Let $\tilde{\alpha}=\left[\alpha^{-}, \alpha^{+}\right] \in[[0,1]]$ and $k \in[-1,0]$ with $\alpha^{+} \leq \mathrm{T}$ and $k \geq \perp$, respectively. Suppose that $\left(M, \mathcal{C}_{(f, \xi)}\right)$ is a crossing cubic subalgebra of $M$. For every $\kappa_{1}, \kappa_{2} \in M$, we have

$$
\begin{aligned}
f_{\tilde{\alpha}}^{T}\left(\kappa_{1} \rightsquigarrow \kappa_{2}\right) & =f\left(\kappa_{1} \rightsquigarrow \kappa_{2}\right)+\tilde{\alpha} \\
& \succeq \operatorname{rmin}\left\{f\left(\kappa_{1}\right), f\left(\kappa_{2}\right)\right\}+\tilde{\alpha} \\
& =\operatorname{rmin}\left\{f\left(\kappa_{1}\right)+\tilde{\alpha}, f\left(\kappa_{2}\right)+\tilde{\alpha}\right\} \\
& =\operatorname{rmin}\left\{f_{\tilde{\alpha}}^{T}\left(\kappa_{1}\right), f_{\tilde{\alpha}}^{T}\left(\kappa_{2}\right)\right\},
\end{aligned}
$$

and

$$
\begin{aligned}
\xi_{\tilde{k}}^{T}\left(\kappa_{1} \rightsquigarrow \kappa_{2}\right) & =\xi\left(\kappa_{1} \rightsquigarrow \kappa_{2}\right)+k \\
& \leq \max \left\{\xi\left(\kappa_{1}\right), \xi\left(\kappa_{2}\right)\right\}+k \\
& =\max \left\{\xi\left(\kappa_{1}\right)+k, \xi\left(\kappa_{2}\right)+k\right\} \\
& =\max \left\{\xi_{k}^{T}\left(\kappa_{1}\right), \xi_{k}^{T}\left(\kappa_{2}\right)\right\}
\end{aligned}
$$

which shows that $\left(M, \mathcal{C}_{\left(f_{\tilde{\alpha}}^{T}, \xi_{k}^{T}\right)}\right)$ is a crossing cubic subalgebra of $M$. Assume that $\left(M, \mathcal{C}_{(f, \xi)}\right)$ is a crossing cubic ideal of $M$ and let $\kappa_{1}, \kappa_{2} \in M$. Then

$$
f_{\tilde{\alpha}}^{T}(0)=f(0)+\tilde{\alpha} \succeq f\left(\kappa_{1}\right)+\tilde{\alpha}=f_{\tilde{\alpha}}^{T}\left(\kappa_{1}\right),
$$

and

$$
\xi_{k}^{T}(0)=\xi(0)+k \leq \xi\left(\kappa_{1}\right)+k=\xi_{k}^{T}\left(\kappa_{1}\right)
$$

Also,

$$
\begin{aligned}
f_{\tilde{\alpha}}^{T}\left(\kappa_{1}\right) & =f\left(\kappa_{1}\right)+\tilde{\alpha} \succeq \operatorname{rmin}\left\{f\left(\kappa_{1} \rightsquigarrow \kappa_{2}\right), f\left(\kappa_{2}\right)\right\}+\tilde{\alpha} \\
& =\operatorname{rmin}\left\{f\left(\kappa_{1} \rightsquigarrow \kappa_{2}\right)+\tilde{\alpha}, f\left(\kappa_{2}\right)+\tilde{\alpha}\right\} \\
& =\operatorname{rmin}\left\{f_{\tilde{\alpha}}^{T}\left(\kappa_{1} \rightsquigarrow \kappa_{2}\right), f_{\tilde{\alpha}}^{T}\left(\kappa_{2}\right)\right\}
\end{aligned}
$$


and

$$
\begin{aligned}
\xi_{k}^{T}\left(\kappa_{1}\right) & =\xi\left(\kappa_{1}\right)+k \leq \max \left\{\xi\left(\kappa_{1} \rightsquigarrow \kappa_{2}\right), \xi\left(\kappa_{2}\right)\right\}+k \\
& =\max \left\{\xi\left(\kappa_{1} \rightsquigarrow \kappa_{2}\right)+k, \xi\left(\kappa_{2}\right)+k\right\} \\
& =\max \left\{\xi_{k}^{T}\left(\kappa_{1} \rightsquigarrow \kappa_{2}\right), \xi_{k}^{T}\left(\kappa_{2}\right)\right\} .
\end{aligned}
$$

Therefore $\left(M, \mathcal{C}_{\left(f_{\tilde{\alpha}}^{T}, \xi_{k}^{T}\right)}\right)$ is a crossing cubic ideal of $M$.

Theorem 4.3. If $\left(M, \mathcal{C}_{(f, \xi)}\right)$ is a crossing cubic o-subalgebra of a BCK-algebra $M$ with the condition (S), then so is its $(\tilde{\alpha}, k)$-translation $\left(M, \mathcal{C}_{\left(f_{\tilde{\alpha}}^{T}, \xi_{k}^{T}\right)}\right)$ for all $\tilde{\alpha}=\left[\alpha^{-}, \alpha^{+}\right] \in[[0,1]]$ and $k \in[-1,0]$ with $\alpha^{+} \leq \mathrm{T}$ and $k \geq \perp$, respectively.

Proof. It is straightforward by the same way to Theorem 4.2 .

Theorem 4.4. If $\left(M, \mathcal{C}_{(f, \xi)}\right)$ is a closed crossing cubic ideal of a BCI-algebra $M$, then so is its $(\tilde{\alpha}, k)$ translation $\left(M, \mathcal{C}_{\left(f_{\tilde{\alpha}}^{T}, \xi_{k}^{T}\right)}\right)$ for all $\tilde{\alpha}=\left[\alpha^{-}, \alpha^{+}\right] \in[[0,1]]$ and $k \in[-1,0]$ with $\alpha^{+} \leq \mathrm{T}$ and $k \geq \perp$, respectively.

Proof. Let $\tilde{\alpha}=\left[\alpha^{-}, \alpha^{+}\right] \in[[0,1]]$ and $k \in[-1,0]$ with $\alpha^{+} \leq \mathrm{T}$ and $k \geq \perp$, respectively. If $\left(M, \mathcal{C}_{(f, \xi)}\right)$ is a closed crossing cubic ideal of a BCI-algebra $M$, then it is a crossing cubic ideal of $M$ and thus its $(\tilde{\alpha}, k)$-translation $\left(M, \mathcal{C}_{\left(f_{\tilde{\alpha}}^{T}, \xi_{k}^{T}\right)}\right)$ is a crossing cubic ideal of $M$ Theorem 4.2 . For every $\kappa_{1} \in M$, we have $f_{\tilde{\alpha}}^{T}\left(0 \rightsquigarrow \kappa_{1}\right)=f\left(0 \rightsquigarrow \kappa_{1}\right)+\tilde{\alpha} \succeq f\left(\kappa_{1}\right)+\tilde{\alpha}=f_{\tilde{\alpha}}^{T}\left(\kappa_{1}\right)$ and $\xi_{k}^{T}\left(0 \rightsquigarrow \kappa_{1}\right)=\xi\left(0 \rightsquigarrow \kappa_{1}\right)+k \leq \xi\left(\kappa_{1}\right)+k=\xi_{k}^{T}\left(\kappa_{1}\right)$. Hence $\left(M, \mathcal{C}_{\left(f_{\tilde{\alpha}}^{T}, \xi_{k}^{T}\right)}\right)$ is a 0 -crossing cubic structure on $M$. It follows from Theorem 3.18 that $\left(M, \mathcal{C}_{\left(f_{\tilde{\alpha}}^{T}, \xi_{k}^{T}\right)}\right)$ is a closed crossing cubic ideal of $M$.

Theorem 4.5. Let $\left(M, \mathcal{C}_{(f, \xi)}\right)$ be a crossing cubic structure on $M$. If there exist $\tilde{\alpha}=\left[\alpha^{-}, \alpha^{+}\right] \in[[0,1]]$ and $k \in[-1,0]$ with $\alpha^{+} \leq \mathrm{T}$ and $k \geq \perp$, respectively, such that the $(\tilde{\alpha}, k)$-translation $\left(M, \mathcal{C}_{\left(f_{\tilde{\alpha}}^{T}, \xi_{k}^{T}\right)}\right)$ of $\left(M, \mathcal{C}_{(f, \xi)}\right)$ is a crossing cubic subalgebra or a crossing cubic ideal of $M$, then $\left(M, \mathcal{C}_{(f, \xi)}\right)$ is a crossing cubic subalgebra or a crossing cubic ideal of $M$.

Proof. Suppose that the $(\tilde{\alpha}, k)$-translation $\left(M, \mathcal{C}_{\left(f_{\tilde{\alpha}}^{T}, \xi_{k}^{T}\right)}\right)$ of $\left(M, \mathcal{C}_{(f, \xi)}\right)$ is a crossing cubic subalgebra of $M$ for some $\tilde{\alpha}=\left[\alpha^{-}, \alpha^{+}\right] \in[[0,1]]$ and $k \in[-1,0]$ with $\alpha^{+} \leq \mathrm{T}$ and $k \geq \perp$, respectively. Then

$$
\begin{aligned}
f\left(\kappa_{1} \rightsquigarrow \kappa_{2}\right)+\tilde{\alpha} & =f_{\tilde{\alpha}}^{T}\left(\kappa_{1} \rightsquigarrow \kappa_{2}\right) \succeq \operatorname{rmin}\left\{f_{\tilde{\alpha}}^{T}\left(\kappa_{1}\right), f_{\tilde{\alpha}}^{T}\left(\kappa_{2}\right)\right\} \\
& =\operatorname{rmin}\left\{f\left(\kappa_{1}\right)+\tilde{\alpha}, f\left(\kappa_{2}\right)+\tilde{\alpha}\right\}=\operatorname{rmin}\left\{f\left(\kappa_{1}\right), f\left(\kappa_{2}\right)\right\}+\tilde{\alpha},
\end{aligned}
$$

and

$$
\begin{aligned}
\xi\left(\kappa_{1} \rightsquigarrow \kappa_{2}\right)+k & =\xi_{k}^{T}\left(\kappa_{1} \rightsquigarrow \kappa_{2}\right) \leq \max \left\{\xi_{k}^{T}\left(\kappa_{1}\right), \xi_{k}^{T}\left(\kappa_{2}\right)\right\} \\
& =\max \left\{\xi\left(\kappa_{1}\right)+k, \xi\left(\kappa_{2}\right)+k\right\}=\max \left\{\xi\left(\kappa_{1}\right), \xi\left(\kappa_{2}\right)\right\}+k .
\end{aligned}
$$

It follows that $f\left(\kappa_{1} \rightsquigarrow \kappa_{2}\right) \succeq \operatorname{rmin}\left\{f\left(\kappa_{1}\right), f\left(\kappa_{2}\right)\right\}$ and $\xi\left(\kappa_{1} \rightsquigarrow \kappa_{2}\right) \leq \max \left\{\xi\left(\kappa_{1}\right), \xi\left(\kappa_{2}\right)\right\}$. Hence $\left(M, \mathcal{C}_{(f, \xi)}\right)$ is a crossing cubic subalgebra of $M$. Now, assume that the $(\tilde{\alpha}, k)$-translation $\left(M, \mathcal{C}_{\left(f_{\tilde{\alpha}}^{T}, \xi_{k}^{T}\right)}\right)$ of $\left(M, \mathcal{C}_{(f, \xi)}\right)$ is a crossing cubic ideal of $M$ for some $\tilde{\alpha}=\left[\alpha^{-}, \alpha^{+}\right] \in[[0,1]]$ and $k \in[-1,0]$ with $\alpha^{+} \leq \mathrm{T}$ and $k \geq \perp$, respectively. Then $f(0)+\tilde{\alpha}=f_{\tilde{\alpha}}^{T}(0) \succeq f_{\tilde{\alpha}}^{T}\left(\kappa_{1}\right)=f\left(\kappa_{1}\right)+\tilde{\alpha}$ and $\xi(0)+k=\xi_{k}^{T}(0) \leq \xi_{k}^{T}\left(\kappa_{1}\right)=\xi\left(\kappa_{1}\right)+k$ for all $\kappa_{1} \in M$. Hence $f(0) \succeq f\left(\kappa_{1}\right)$ and $\xi(0) \leq \xi\left(\kappa_{1}\right)$ for all $\kappa_{1} \in M$. Also, we have

$$
\begin{aligned}
f\left(\kappa_{1}\right)+\tilde{\alpha} & =f_{\tilde{\alpha}}^{T}\left(\kappa_{1}\right) \succeq \operatorname{rmin}\left\{f_{\tilde{\alpha}}^{T}\left(\kappa_{1} \rightsquigarrow \kappa_{2}\right), f_{\tilde{\alpha}}^{T}\left(\kappa_{2}\right)\right\} \\
& =\operatorname{rmin}\left\{f\left(\kappa_{1} \rightsquigarrow \kappa_{2}\right)+\tilde{\alpha}, f\left(\kappa_{2}\right)+\tilde{\alpha}\right\} \\
& =\operatorname{rmin}\left\{f\left(\kappa_{1} \rightsquigarrow \kappa_{2}\right), f\left(\kappa_{2}\right)\right\}+\tilde{\alpha},
\end{aligned}
$$


and

$$
\begin{aligned}
\xi\left(\kappa_{1}\right)+k & =\xi_{k}^{T}\left(\kappa_{1}\right) \leq \max \left\{\xi_{k}^{T}\left(\kappa_{1} \rightsquigarrow \kappa_{2}\right), \xi_{k}^{T}\left(\kappa_{2}\right)\right\} \\
& =\max \left\{\xi\left(\kappa_{1} \rightsquigarrow \kappa_{2}\right)+k, \xi\left(\kappa_{2}\right)+k\right\} \\
& =\max \left\{\xi\left(\kappa_{1} \rightsquigarrow \kappa_{2}\right), \xi\left(\kappa_{2}\right)\right\}+k,
\end{aligned}
$$

which imply that $f\left(\kappa_{1}\right) \succeq \operatorname{rmin}\left\{f\left(\kappa_{1} \rightsquigarrow \kappa_{2}\right), f\left(\kappa_{2}\right)\right\}$ and $\xi\left(\kappa_{1}\right) \leq \max \left\{\xi\left(\kappa_{1} \rightsquigarrow \kappa_{2}\right), \xi\left(\kappa_{2}\right)\right\}$ for all $\kappa_{1}, \kappa_{2} \in M$. Consequently, $\left(M, \mathcal{C}_{(f, \xi)}\right)$ is a crossing cubic ideal of $M$.

The following results are derived in the same way.

Theorem 4.6. Let $\left(M, \mathcal{C}_{(f, \xi)}\right)$ be a crossing cubic structure on a BCK-algebra $M$ with the condition (S). If there exist $\tilde{\alpha}=\left[\alpha^{-}, \alpha^{+}\right] \in[[0,1]]$ and $k \in[-1,0]$ with $\alpha^{+} \leq \mathrm{T}$ and $k \geq \perp$, respectively, such that the $(\tilde{\alpha}, k)$-translation $\left(M, \mathcal{C}_{\left(f_{\tilde{\alpha}}^{T}, \xi_{k}^{T}\right)}\right)$ of $\left(M, \mathcal{C}_{(f, \xi)}\right)$ is a crossing cubic o-subalgebra of $M$, then $\left(M, \mathcal{C}_{(f, \xi)}\right)$ is a crossing cubic o-subalgebra of $M$.

Theorem 4.7. Let $\left(M, \mathcal{C}_{(f, \xi)}\right)$ be a crossing cubic structure on a BCI-algebra $M$. If there exist $\tilde{\alpha}=$ $\left[\alpha^{-}, \alpha^{+}\right] \in[[0,1]]$ and $k \in[-1,0]$ with $\alpha^{+} \leq \mathrm{T}$ and $k \geq \perp$, respectively, such that the $(\tilde{\alpha}, k)$-translation $\left(M, \mathcal{C}_{\left(f_{\tilde{\alpha}}^{T}, \xi_{k}^{T}\right)}\right)$ of $\left(M, \mathcal{C}_{(f, \xi)}\right)$ is a closed crossing cubic ideal of $M$, then $\left(M, \mathcal{C}_{(f, \xi)}\right)$ is a closed crossing cubic ideal of $M$.

Let $\left(M, \mathcal{C}_{(f, \xi)}\right)$ be a crossing cubic structure on $M$ and consider $\tilde{\alpha}=\left[\alpha^{-}, \alpha^{+}\right] \in[[0,1]]$ and $k \in[-1,0]$ with $\alpha^{+} \leq \mathrm{T}$ and $k \geq \perp$, respectively. We take a set

$$
\mathcal{C}_{(f, \xi)}(\tilde{\beta}, r):=\left\{\kappa_{1} \in M \mid f\left(\kappa_{1}\right) \succeq \tilde{\beta}-\tilde{\alpha}, \xi\left(\kappa_{1}\right) \leq r-k\right\}
$$

where $\tilde{\beta} \in[[0,1]]$ and $r \in[-1,0]$ such that $\tilde{\beta} \succ \tilde{\alpha}, \beta^{+} \leq \mathrm{T}$ and $r<k$.

Theorem 4.8. If $\left(M, \mathcal{C}_{(f, \xi)}\right)$ is a crossing cubic subalgebra or a crossing cubic ideal of $M$, then the set $\mathcal{C}_{(f, \xi)}(\tilde{\beta}, r)$ in $[33)$ is a subalgebra or an ideal of $M$ for all $\tilde{\beta} \in[[0,1]]$ and $r \in[-1,0]$ such that $\tilde{\beta} \succ \tilde{\alpha}$, $\beta^{+} \leq \mathrm{T}$ and $r<k$.

Proof. Let $\tilde{\beta} \in[[0,1]]$ and $r \in[-1,0]$ be such that $\tilde{\beta} \succ \tilde{\alpha}, \beta^{+} \leq \mathrm{T}$ and $r<k$. Assume that $\left(M, \mathcal{C}_{(f, \xi)}\right)$ is a crossing cubic subalgebra of $M$. Let $\kappa_{1}, \kappa_{2} \in \mathcal{C}_{(f, \xi)}(\tilde{\beta}, r)$. Then $f\left(\kappa_{1}\right) \succeq \tilde{\beta}-\tilde{\alpha}, \xi\left(\kappa_{1}\right) \leq r-k$, $f\left(\kappa_{2}\right) \succeq \tilde{\beta}-\tilde{\alpha}$, and $\xi\left(\kappa_{2}\right) \leq r-k$. Hence $f\left(\kappa_{1} \rightsquigarrow \kappa_{2}\right) \succeq \operatorname{rmin}\left\{f\left(\kappa_{1}\right), f\left(\kappa_{2}\right)\right\} \succeq \tilde{\beta}-\tilde{\alpha}$ and $\xi\left(\kappa_{1} \rightsquigarrow \kappa_{2}\right) \leq$ $\max \left\{\xi\left(\kappa_{1}\right), \xi\left(\kappa_{2}\right)\right\} \leq r-k$. Thus $\kappa_{1} \rightsquigarrow \kappa_{2} \in \mathcal{C}_{(f, \xi)}(\tilde{\beta}, r)$, and so $\mathcal{C}_{(f, \xi)}(\tilde{\beta}, r)$ is a subalgebra of $M$. Now, suppose that $\left(M, \mathcal{C}_{(f, \xi)}\right)$ is a crossing cubic ideal of $M$ and let $\kappa_{1}, \kappa_{2} \in M$ be such that $\kappa_{1} \rightsquigarrow \kappa_{2} \in \mathcal{C}_{(f, \xi)}(\tilde{\beta}, r)$ and $\kappa_{2} \in \mathcal{C}_{(f, \xi)}(\tilde{\beta}, r)$. Then $f\left(\kappa_{1} \rightsquigarrow \kappa_{2}\right) \succeq \tilde{\beta}-\tilde{\alpha}, \xi\left(\kappa_{1} \rightsquigarrow \kappa_{2}\right) \leq r-k, f\left(\kappa_{2}\right) \succeq \tilde{\beta}-\tilde{\alpha}$, and $\xi\left(\kappa_{2}\right) \leq r-k$. It follows that $f\left(\kappa_{1}\right) \succeq \operatorname{rmin}\left\{f\left(\kappa_{1} \rightsquigarrow \kappa_{2}\right), f\left(\kappa_{2}\right)\right\} \succeq \tilde{\beta}-\tilde{\alpha}$ and $\xi\left(\kappa_{1}\right) \leq \max \left\{\xi\left(\kappa_{1} \rightsquigarrow \kappa_{2}\right), \xi\left(\kappa_{2}\right)\right\} \leq r-k$. Thus $\kappa_{1} \in \mathcal{C}_{(f, \xi)}(\tilde{\beta}, r)$. It is clear that $0 \in \mathcal{C}_{(f, \xi)}(\tilde{\beta}, r)$. Therefore $\mathcal{C}_{(f, \xi)}(\tilde{\beta}, r)$ is an ideal of $M$.

We find conditions for the $(\tilde{\alpha}, k)$-translation of a crossing cubic structure on $M$ to be a crossing cubic subalgebra (ideal) of $M$.

Theorem 4.9. Let $\left(M, \mathcal{C}_{(f, \xi)}\right)$ be a crossing cubic structure on $M$. Then the $(\tilde{\alpha}, k)$-translation $\left(M, \mathcal{C}_{\left(f_{\tilde{\alpha}}^{T}, \xi_{k}^{T}\right)}\right)$ of $\left(M, \mathcal{C}_{(f, \xi)}\right)$ is a crossing cubic subalgebra or a crossing cubic ideal of $M$ if and only if the nonempty sets

$$
\mathcal{C}_{(f, \xi)}(\tilde{\beta}):=\left\{\kappa_{1} \in M \mid f\left(\kappa_{1}\right) \succeq \tilde{\beta}-\tilde{\alpha}\right\}, \text { and } \mathcal{C}_{(f, \xi)}(r):=\left\{\kappa_{1} \in M \mid \xi\left(\kappa_{1}\right) \leq r-k\right\},
$$

are subalgebras or ideals of $M$ for all $\tilde{\beta} \in[[0,1]]$ and $r \in[-1,0]$ such that $\tilde{\beta} \succ \tilde{\alpha}, \beta^{+} \leq \mathrm{T}$ and $r<k$.

Proof. Suppose that the $(\tilde{\alpha}, k)$-translation $\left(M, \mathcal{C}_{\left(f_{\tilde{\alpha}}^{T}, \xi_{k}^{T}\right)}\right)$ of $\left(M, \mathcal{C}_{(f, \xi)}\right)$ is a crossing cubic subalgebra of $M$. Let $\kappa_{1}, \kappa_{2} \in \mathcal{C}_{(f, \xi)}(\tilde{\beta})$ and $a, b \in \mathcal{C}_{(f, \xi)}(r)$ for all $\tilde{\beta} \in[[0,1]]$ and $r \in[-1,0]$ such that $\tilde{\beta} \succ \tilde{\alpha}, \beta^{+} \leq \mathrm{T}$ and 
$r<k$. Then $f\left(\kappa_{1}\right) \succeq \tilde{\beta}-\tilde{\alpha}, f\left(\kappa_{2}\right) \succeq \tilde{\beta}-\tilde{\alpha}, \xi(a) \leq r-k$, and $\xi(b) \leq r-k$. It follows that

$$
\begin{aligned}
f\left(\kappa_{1} \rightsquigarrow \kappa_{2}\right)+\tilde{\alpha} & =f_{\tilde{\alpha}}^{T}\left(\kappa_{1} \rightsquigarrow \kappa_{2}\right) \succeq \operatorname{rmin}\left\{f_{\tilde{\alpha}}^{T}\left(\kappa_{1}\right), f_{\tilde{\alpha}}^{T}\left(\kappa_{2}\right)\right\} \\
& =\operatorname{rmin}\left\{f\left(\kappa_{1}\right)+\tilde{\alpha}, f\left(\kappa_{2}\right)+\tilde{\alpha}\right\} \\
& =\operatorname{rmin}\left\{f\left(\kappa_{1}\right), f\left(\kappa_{2}\right)\right\}+\tilde{\alpha} \\
& \succeq \tilde{\beta}-\tilde{\alpha}+\tilde{\alpha}=\tilde{\beta},
\end{aligned}
$$

and

$$
\begin{aligned}
\xi(a \rightsquigarrow b)+k & =\xi_{k}^{T}(a \rightsquigarrow b) \leq \max \left\{\xi_{k}^{T}(a), \xi_{k}^{T}(b)\right\} \\
& =\max \{\xi(a)+k, \xi(b)+k\} \\
& =\max \{\xi(a), \xi(b)\}+k \\
& \leq r-k+k=r .
\end{aligned}
$$

Hence $f\left(\kappa_{1} \rightsquigarrow \kappa_{2}\right) \succeq \tilde{\beta}-\tilde{\alpha}$ and $\xi(a \rightsquigarrow b) \leq r-k$, which shows that $\kappa_{1} \rightsquigarrow \kappa_{2} \in \mathcal{C}_{(f, \xi)}(\tilde{\beta})$ and $a \rightsquigarrow b \in \mathcal{C}_{(f, \xi)}(r)$. Thus $\mathcal{C}_{(f, \xi)}(\tilde{\beta})$ and $\mathcal{C}_{(f, \xi)}(r)$ are subalgebras of $M$. Assume that the $\left(M, \mathcal{C}_{\left(f_{\tilde{\alpha}}^{T}, \xi_{k}^{T}\right)}\right)$ is a crossing cubic ideal of $M$. Let $\kappa_{1} \in \mathcal{C}_{(f, \xi)}(\tilde{\beta})$ and $a \in \mathcal{C}_{(f, \xi)}(r)$ for all $\tilde{\beta} \in[[0,1]]$ and $r \in[-1,0]$ such that $\tilde{\beta} \succ \tilde{\alpha}, \beta^{+} \leq \mathrm{T}$ and $r<k$. Then

$$
f(0)+\tilde{\alpha}=f_{\tilde{\alpha}}^{T}(0) \succeq f_{\tilde{\alpha}}^{T}\left(\kappa_{1}\right)=f\left(\kappa_{1}\right)+\tilde{\alpha} \succeq \tilde{\beta}-\tilde{\alpha}+\tilde{\alpha}=\tilde{\beta},
$$

and

$$
\xi(0)+k=\xi_{k}^{T}(0) \leq \xi_{k}^{T}\left(\kappa_{1}\right)=\xi\left(\kappa_{1}\right)+k \leq r-k+k=r,
$$

which shows that $0 \in \mathcal{C}_{(f, \xi)}(\tilde{\beta}) \cap \mathcal{C}_{(f, \xi)}(r)$. Let $\kappa_{1}, \kappa_{2}, a, b \in M$ be such that $\kappa_{1} \rightsquigarrow \kappa_{2} \in \mathcal{C}_{(f, \xi)}(\tilde{\beta}), \kappa_{2} \in$ $\mathcal{C}_{(f, \xi)}(\tilde{\beta}), a \rightsquigarrow b \in \mathcal{C}_{(f, \xi)}(r)$ and $b \in \mathcal{C}_{(f, \xi)}(r)$. Then

$$
\begin{aligned}
f\left(\kappa_{1}\right)+\tilde{\alpha} & =f_{\tilde{\alpha}}^{T}\left(\kappa_{1}\right) \succeq \operatorname{rmin}\left\{f_{\tilde{\alpha}}^{T}\left(\kappa_{1} \rightsquigarrow \kappa_{2}\right), f_{\tilde{\alpha}}^{T}\left(\kappa_{2}\right)\right\} \\
& =\operatorname{rmin}\left\{f\left(\kappa_{1} \rightsquigarrow \kappa_{2}\right)+\tilde{\alpha}, f\left(\kappa_{2}\right)+\tilde{\alpha}\right\} \\
& =\operatorname{rmin}\left\{f\left(\kappa_{1} \rightsquigarrow \kappa_{2}\right), f\left(\kappa_{2}\right)\right\}+\tilde{\alpha} \\
& \succeq \tilde{\beta}-\tilde{\alpha}+\tilde{\alpha}=\tilde{\beta},
\end{aligned}
$$

and

$$
\begin{aligned}
\xi(a)+k & =\xi_{k}^{T}(a) \leq \max \left\{\xi_{k}^{T}(a \rightsquigarrow b), \xi_{k}^{T}(b)\right\} \\
& =\max \{\xi(a \rightsquigarrow b)+k, \xi(b)+k\} \\
& =\max \{\xi(a \rightsquigarrow b), \xi(b)\}+k \\
& \leq r-k+k=r .
\end{aligned}
$$

Hence $\kappa_{1} \in \mathcal{C}_{(f, \xi)}(\tilde{\beta})$ and $a \in \mathcal{C}_{(f, \xi)}(r)$. Therefore $\mathcal{C}_{(f, \xi)}(\tilde{\beta})$ and $\mathcal{C}_{(f, \xi)}(r)$ are ideals of $M$.

Conversely, suppose that $\mathcal{C}_{(f, \xi)}(\tilde{\beta})$ and $\mathcal{C}_{(f, \xi)}(r)$ are subalgebras of $M$ for all $\tilde{\beta} \in[[0,1]]$ and $r \in[-1,0]$ such that $\tilde{\beta} \succ \tilde{\alpha}, \beta^{+} \leq \mathrm{T}$ and $r<k$. If there exist $(a, b),\left(\kappa_{1}, \kappa_{2}\right) \in M \times M$ such that $f_{\tilde{\alpha}}^{T}(a \rightsquigarrow b) \succeq$ $\operatorname{rmin}\left\{f_{\tilde{\alpha}}^{T}(a), f_{\tilde{\alpha}}^{T}(b)\right\}$ and $\xi_{k}^{T}\left(\kappa_{1} \rightsquigarrow \kappa_{2}\right) \leq \max \left\{\xi_{k}^{T}\left(\kappa_{1}\right), \xi_{k}^{T}\left(\kappa_{2}\right)\right\}$ are not true respectively, then $f_{\tilde{\alpha}}^{T}(a \rightsquigarrow b) \prec$ $\tilde{\varepsilon} \preceq \operatorname{rmin}\left\{f_{\tilde{\alpha}}^{T}(a), f_{\tilde{\alpha}}^{T}(b)\right\}$ and $\xi_{k}^{T}\left(\kappa_{1} \rightsquigarrow \kappa_{2}\right)>s \geq \max \left\{\xi_{k}^{T}\left(\kappa_{1}\right), \xi_{k}^{T}\left(\kappa_{2}\right)\right\}$ for some $\tilde{\varepsilon} \in[[0,1]]$ and $s \in[-1,0]$ such that $\tilde{\varepsilon} \succ \tilde{\alpha}, \varepsilon^{+} \leq \mathrm{T}$ and $s<k$. It follows that $f(a) \succeq \tilde{\varepsilon}-\tilde{\alpha}, f(b) \succeq \tilde{\varepsilon}-\tilde{\alpha}, \xi\left(\kappa_{1}\right) \leq s-k$ and $\xi\left(\kappa_{2}\right) \leq s-k$, that is, $a, b \in \mathcal{C}_{(f, \xi)}(\tilde{\beta})$ and $\kappa_{1}, \kappa_{2} \in \mathcal{C}_{(f, \xi)}(r)$. But $f(a \rightsquigarrow b) \nsucceq \tilde{\varepsilon}-\tilde{\alpha}$ and $\xi\left(\kappa_{1} \rightsquigarrow \kappa_{2}\right) \not \leq s-k$, that is, $a \rightsquigarrow b \notin \mathcal{C}_{(f, \xi)}(\tilde{\beta})$ and $\kappa_{1} \rightsquigarrow \kappa_{2} \notin \mathcal{C}_{(f, \xi)}(r)$. This is a contradiction, and thus $f_{\tilde{\alpha}}^{T}\left(\kappa_{1} \rightsquigarrow \kappa_{2}\right) \succeq$ $\operatorname{rmin}\left\{f_{\tilde{\alpha}}^{T}\left(\kappa_{1}\right), f_{\tilde{\alpha}}^{T}\left(\kappa_{2}\right)\right\}$ and $\xi_{k}^{T}\left(\kappa_{1} \rightsquigarrow \kappa_{2}\right) \leq \max \left\{\xi_{k}^{T}\left(\kappa_{1}\right), \xi_{k}^{T}\left(\kappa_{2}\right)\right\}$. Hence $\left(M, \mathcal{C}_{\left(f_{\tilde{\alpha}}^{T}, \xi_{k}^{T}\right)}\right)$ is a crossing cubic subalgebra of $M$. 
Assume that $\mathcal{C}_{(f, \xi)}(\tilde{\beta})$ and $\mathcal{C}_{(f, \xi)}(r)$ are ideals of $M$ for all $\tilde{\beta} \in[[0,1]]$ and $r \in[-1,0]$ such that $\tilde{\beta} \succ \tilde{\alpha}$, $\beta^{+} \leq \mathrm{T}$ and $r<k$. If there exists $\left(a, \kappa_{1}\right) \in M \times M$ such that $f_{\tilde{\alpha}}^{T}(0) \succeq f_{\tilde{\alpha}}^{T}(a)$ and $\xi_{k}^{T}(0) \leq \xi_{k}^{T}\left(\kappa_{1}\right)$ are not true respectively, then $f_{\tilde{\alpha}}^{T}(0) \prec \tilde{\varepsilon} \preceq f_{\tilde{\alpha}}^{T}(a)$ and $\xi_{k}^{T}(0)>s \geq \xi_{k}^{T}\left(\kappa_{1}\right)$ for some $\tilde{\varepsilon} \in[[0,1]]$ and $s \in[-1,0]$ such that $\tilde{\varepsilon} \succ \tilde{\alpha}, \varepsilon^{+} \leq \mathrm{T}$ and $s<k$. Then $0 \notin \mathcal{C}_{(f, \xi)}(\tilde{\beta}) \cap \mathcal{C}_{(f, \xi)}(r)$, which is a contradiction and so $f_{\tilde{\alpha}}^{T}(0) \succeq f_{\tilde{\alpha}}^{T}\left(\kappa_{1}\right)$ and $\xi_{k}^{T}(0) \leq \xi_{k}^{T}\left(\kappa_{1}\right)$ for all $\kappa_{1} \in M$. If there exist $(a, b),\left(\kappa_{1}, \kappa_{2}\right) \in M \times M$ such that $f_{\tilde{\alpha}}^{T}(a) \succeq \operatorname{rmin}\left\{f_{\tilde{\alpha}}^{T}(a \rightsquigarrow b), f_{\tilde{\alpha}}^{T}(b)\right\}$ and $\xi_{k}^{T}\left(\kappa_{1}\right) \leq \max \left\{\xi_{k}^{T}\left(\kappa_{1} \rightsquigarrow \kappa_{2}\right), \xi_{k}^{T}\left(\kappa_{2}\right)\right\}$ are not true respectively, then $f_{\tilde{\alpha}}^{T}(a) \prec \tilde{\varepsilon} \preceq \operatorname{rmin}\left\{f_{\tilde{\alpha}}^{T}(a \rightsquigarrow b), f_{\tilde{\alpha}}^{T}(b)\right\}$ and $\xi_{k}^{T}\left(\kappa_{1}\right)>s \geq \max \left\{\xi_{k}^{T}\left(\kappa_{1} \rightsquigarrow \kappa_{2}\right), \xi_{k}^{T}\left(\kappa_{2}\right)\right\}$ for some $\tilde{\varepsilon} \in[[0,1]]$ and $s \in[-1,0]$ such that $\tilde{\varepsilon} \succ \tilde{\alpha}, \varepsilon^{+} \leq \mathrm{T}$ and $s<k$. It follows that $f(a \rightsquigarrow b) \succeq \tilde{\varepsilon}-\tilde{\alpha}, f(b) \succeq \tilde{\varepsilon}-\tilde{\alpha}$, $\xi\left(\kappa_{1} \rightsquigarrow \kappa_{2}\right) \leq s-k$ and $\xi\left(\kappa_{2}\right) \leq s-k$, that is, $a \rightsquigarrow b, b \in \mathcal{C}_{(f, \xi)}(\tilde{\beta})$ and $\kappa_{1} \rightsquigarrow \kappa_{2}, \kappa_{2} \in \mathcal{C}_{(f, \xi)}(r)$. But $f(a) \nsucceq \tilde{\varepsilon}-\tilde{\alpha}$ and $\xi\left(\kappa_{1}\right) \not \leq s-k$, that is, $a \notin \mathcal{C}_{(f, \xi)}(\tilde{\beta})$ and $\kappa_{1} \notin \mathcal{C}_{(f, \xi)}(r)$. This is a contradiction, and thus $f_{\tilde{\alpha}}^{T}\left(\kappa_{1}\right) \succeq \operatorname{rmin}\left\{f_{\tilde{\alpha}}^{T}\left(\kappa_{1} \rightsquigarrow \kappa_{2}\right), f_{\tilde{\alpha}}^{T}\left(\kappa_{2}\right)\right\}$ and $\xi_{k}^{T}\left(\kappa_{1}\right) \leq \max \left\{\xi_{k}^{T}\left(\kappa_{1} \rightsquigarrow \kappa_{2}\right), \xi_{k}^{T}\left(\kappa_{2}\right)\right\}$ for all $\kappa_{1}, \kappa_{2} \in M$. Therefore $\left(M, \mathcal{C}_{\left(f_{\bar{\alpha}}^{T}, \xi_{k}^{T}\right)}\right)$ is a crossing cubic ideal of $M$.

\section{Conclusions}

Jun et al. introduced the notion of crossing cubic structures by using the negative-valued function and interval-valued fuzzy set, and then they applied it to BCK/BCI-algebras. In this manuscript, we have introduced the notions of a crossing cubic ideal in a BCK/BCI-algebra, a closed crossing cubic ideal in a BCIalgebra, and a crossing cubic o-subalgebra of a BCK-algebra with the condition (S). We have identified the relationship between them, and have provided conditions for a crossing cubic structure to be a closed crossing cubic ideal. We have explored the conditions under which crossing cubic ideals are closed, and have discussed characterizations of crossing cubic ideals. We have studied the translation of crossing cubic subalgebras and crossing cubic ideals, and provided conditions for the translation of a crossing cubic structure to be a crossing cubic subalgebra (ideal), and then we have considered its characterization. In [1, Borzooei et al. discussed several results on hoops. They studied relations between hoops and some logical algebras. Recently, Mohseni Takallo et al. 8] studied neutrosophic set theory in equality algebras. Going forward, we will study the substructures of several algebraic structures, including hoops and equality algebras, based on the ideas and results of this paper.

\section{References}

[1] R.A. Borzooei, M. Aaly Kologani, Results on hoops, Journal of Algebraic Hyperstructures and Logical Algebras, 1(1) (2020), 61-77.

[2] Y. Huang, BCI-Algebra, Science Press: Beijing, China, 2006.

[3] Y.B. Jun, K. Hur, J.G. Lee, J. Kim, Crossing cubic structures as an extension of bipolar fuzzy sets, Annals of Fuzzy Mathematics and Informatics, in press.

[4] Y.B. Jun, K.J. Lee, S.Z. Song, $\mathcal{N}$-ideals of BCK/BCI-algebras, Journal of the Chungcheong Mathematical Society, 22 (2009), 417-437.

[5] K.M. Lee, Bipolar-valued fuzzy sets and their operations, Proc. Int. Conf. on Intelligent Technologies, Bangkok, Thailand, (2000), 307-312.

[6] J. Meng, Y.B. Jun, BCK-Algebras, Kyung Moon Sa Co.: Seoul, Korea, 1994.

[7] J. Meng, S.M. Wei, Periods of elements in BCI-algebras, Mathematica Japan, 38 (1993), 427-431.

[8] M. Mohseni Takallo, M. Aaly Kologani, MBJ-neutrosophic filters of equality algebras, Journal of Algebraic Hyperstructures and Logical Algebras, 1(2) (2020), 57-75.

[9] L.A. Zadeh, Fuzzy sets, Information and Control, 8 (1965), 338-353.

[10] L.A. Zadeh, The concept of a linguistic variable and its application to approximate reasoning-I, Information Sciences, 8 (1975), 199-249. 